\title{
IMPROVED PHYSICAL MODELS FOR ADVANCED SILICON DEVICE PROCESSING
}

Lourdes Pelaz, Luis A. Marqués, María Aboy, Pedro López, and Iván Santos

Departamento de Electrónica, Universidad de Valladolid, E.T.S.I. de Telecomunicación, 47011 Valladolid, SPAIN.

\begin{abstract}
We review atomistic modeling approaches for issues related to ion implantation and annealing in advanced device processing. We describe how models have been upgraded to capture physical mechanisms in more detail as a response to the accuracy demanded in modern process and device modeling. Implantation and damage models based on the binary collision approximation have been improved to describe the direct formation of amorphous pockets for heavy or molecular ions. The use of amorphizing implants followed by solid phase epitaxial regrowth has motivated the development of detailed models that account for amorphization and recrystallization, considering the influence of crystal orientation and stress conditions. We apply simulations to describe the role of implant parameters to minimize residual damage, and we address doping issues that arise in non-planar structures such as FinFETs.
\end{abstract}

Keywords: silicon, ion implantation, annealing, atomistic simulation. 


\section{Introduction}

The continuous scaling down of electronic devices has revealed the necessity of innovative nanostructures to overtake the limitations of traditional planar MOSFETs in present and future technological nodes [1]. Thus, a change from the traditional 2D planar FETs to 3D multigate MOS devices has been imposed to improve device performance [2-5]. FinFETs [6-8] are already in production at industrial level, while the use of gateall-around devices [9-11] and nanowire FETs [12-15] is being considered for near-future technology nodes as a promising scaling approach.

Ion implantation is still the most common technique used to selectively introduce dopants in planar and multigate FET devices for junction formation. This technology is also being used for pocket formation, threshold-voltage or work-function adjustment in advanced memory and CMOS devices [16]. Power devices, CMOS image sensors and even solar cells [17] also take advantage of the precise control in the dopant distribution provided by ion implantation. However, as the energetic incoming ions penetrate into the substrate, the crystal lattice becomes damaged. Subsequent thermal anneals are required to heal the crystal defects and to electrically activate dopants. The compromise to remove damage and to control dopant activation and diffusion has driven sophisticated implantation and annealing schemes. Modern devices involve a mixture of implant species, cold and hot implants followed by processing steps at low temperatures $\left(<650^{\circ} \mathrm{C}\right)$ combined with high temperature anneals with rapid temperature-time variations (flash and laser anneals) $[18,19]$.

Semiconductor technology faces up to continuous challenges to satisfy the requirements of scalability of new devices and low power/high performance. The minimization of defects is a pressing concern in all devices because they are responsible for increased leakage currents, carrier mobility degradation and enhanced dopant diffusivity, among other deleterious effects [20-22]. Variability is also crucial in modern devices compared to past technologies. It is associated to inaccuracy in angle, energy, dose or temperature control during implants, wafer temperature variations, random dopant fluctuations or inhomogeneous defect distributions. In addition, reduced sizes and complex geometries of nanoscale devices introduce additional complexity in the characterization techniques [23]. 
The fabrication and design of new integrated circuits makes the use of technology computer-aided design tools essential to reduce the development times and costs [24]. In fact, trends in device technology drive the requirements for process modeling. Simulation capabilities evolve to encompass processes, geometries and materials considered for present and future devices. Scaling of transistors does not only demand a higher degree of accuracy but also may require new models for effects that were neglected in previous technology nodes. As the classical Si scaling is reaching its limits and many options are being explored (tunneling devices, quantum electronics, etc. [25]), physics based models should provide understanding and predictive capabilities that could guide technology.

The mainstream tool at industrial level for the simulation and modeling of ion implantation and annealing processes in Si relies on semi-empirical analytical expressions for implanted dopant and defect profiles, and on continuum models in which interactions among dopants and defects are formulated as a series of coupled partial differential equations [24,26]. For enough accuracy and predictability, many interactions should be considered which involve numerous rate equations and unknown parameters. In addition, the presence of very large concentration gradients, high extrinsic doping levels, dopant clustering, dopant segregation, impurities such as C, F or N, amorphous-crystalline (a/c) phase transitions, heavily strained Si and SiGe, and 3D device geometries make this approach difficult and not very efficient.

In this scenario atomistic modeling is gaining relevance in process simulation. Atomistic methods are time consuming as they consider interactions at atomic level, however they are more flexible when applied to systems that require a detailed description or involve complex materials and device architectures. The advances in the understanding of the physics behind dopant-defect interactions and the explosion of computer resources (large memory cells and multi-processor computers) at an affordable price have greatly contributed to the progress in atomistic process simulation. The reduction of device size and the increase of computer power have enabled full atomistic simulation of real structures. Kinetic Monte Carlo (KMC) models [27,28] are beginning to play an important role, both for fundamental research and as a pathway to improve continuum models, and also for direct process simulation at industrial level [24]. KMC methods are based on the random selection of events and particles (dopants and defects) which are relevant for the particular material and process to be simulated. The probability of occurrence of every event is determined by an activation energy and a pre-exponential 
factor. Once all events have been assigned a probability, a random number determines which particular event will take place and which particle will be involved. Then, the simulation time is increased accordingly to the selected event frequency. KMC models make use of a large number of parameters which are usually extracted from experiments or more fundamental simulation techniques, such as classical molecular dynamics (CMD) $[29,30]$ or ab-initio methods [31,32].

The prominence of $\mathrm{Si}$ in electronic circuits has driven intense research on this material over the past decades, leading to modeling capabilities with a high sophistication level. Nevertheless, existing understanding is still improving, some issues remain unsolved and new challenges are appearing. For instance, just recently unexpected $\{001\}$ dislocation loops have been observed in laser annealed implanted Si [33]. Defect growth models based on coalescence instead of conventional Ostwald ripening have been proposed to account for such defects [30]. Other materials that are only recently being considered for integration into mainstream devices, such as $\mathrm{Ge}$ and compound semiconductors (III-V's, SiC), have not been researched as much as $\mathrm{Si}$, and there is a lack of understanding even of fundamental parameters and processes [34]. In principle, research on new materials can be built on the experiences and methods developed for Si. But even for Ge, so similar to Si in many aspects, the extrapolation of Si models is not straightforward [35].

In this paper we review atomistic process modeling approaches for issues related to ion implantation in novel devices. We go into detail about the evolution of implantation and damage models from approximate empirical descriptions to detailed atomistic models in response to the accuracy demanded in modern process technology. We apply atomistic simulations to describe the role of implant parameters to minimize residual damage and we address doping issues that arise in non-planar structures.

\section{Towards a comprehensive description of ion-implanted damage}

Ion implantation has been used in Si technology in a routine way. For many years, analytic distributions were used to obtain fast estimations of dopant and damage depth profiles [36]. These profiles were characterized in terms of the projected range and straggling of implanted ions and generated damage. The parameters that define these distributions need to be calibrated for each set of implantation conditions (ion type, 
energy, incident angle, etc.) Thus, they cannot be used to predict dopant profiles for implantations that have not being previously tabulated. In addition, they do not provide any information at the atomic level of the damage generated during the implantation process. This information is essential to account for dynamic annealing or local inhomogeneities.

Monte Carlo methods based on the binary collision approximation (BCA) overcome this limitation as they provide an atomistic description of individual collision cascades during the implantation process. The basic idea of BCA is that energetic moving ions that penetrate the implanted target only collide with their closest target atom. These collisions can be numerically solved from the energy and momentum conservation laws [37-39]. The target atoms are placed in crystalline lattice sites (such as in MARLOWE $[37,40,41]$ and IIS [42] codes) or in random positions to reproduce an amorphous material (such as in SRIM code [43]). This has consequences in the channeling of the implanted ions and therefore in the tails of the profiles. In any case, a target atom is displaced from its lattice position when its energy after the collision exceeds the displacement energy threshold, $E_{d}$ [37]. For Si, experimental and theoretical estimations for $E_{d}$ range from 10 to $30 \mathrm{eV}$ [44-46], but for most BCA simulators it is conventionally taken as $15 \mathrm{eV}[40,43]$. For energy transfers above $E_{d}$, the target atom can become a recoil, leaving behind a vacancy and generating a Si self-interstitial where it stops. For energy transfers below $E_{d}$ the target atom is not displaced and the energy is assumed to be lost to phonons. This treatment of implantation cascades allows simulating almost any implantation condition without additional calibrations.

Some BCA simulators only track the trajectory of the implanted ion, and the number of displaced atoms $N_{D A}$ is estimated taking into account the energy deposited into a given target volume $E_{\text {dep }}$ using the modified Kinchin-Pease formula [47]:

$$
N_{D A}=0.42 \frac{E_{d e p}}{E_{d}}
$$

Within this approach [48], ion profiles are rapidly evaluated and generally agree with experiments. The depth distribution of dopants and defects is obtained by simulating a large number of implant cascades, but at the expense of losing the atomic description of damage. 
To obtain the complete description of the damage generated within BCA, it is necessary to follow the trajectories of the implanted ion and all the generated recoils (full cascade BCA). The result of this type of simulation is the position of the implanted ion and the generated Si self-interstitials and vacancies, called Frenkel pairs (FPs), which can be the input for KMC codes. This approach adequately reproduces the dilute damage generated by light ions. However, it is not able to reproduce more complex damage and amorphous structures observed experimentally for heavy ions or molecular implants. These implant conditions have to be properly modeled since ions such as Ge or As are frequently used in the manufacturing of Si devices as preamorphizing steps [49,50], and molecular implants with octadecaborane are considered as an alternative to monatomic B implants because they are self-amorphizing and minimize residual damage [51].

In order to obtain a detailed and accurate description of damage structures generated during cascades it is necessary to resort to CMD simulations. In CMD the interactions among atoms are described through an empirical potential, which is an analytic function developed and fitted to reproduce the equilibrium properties of the simulated material (crystal structure, lattice constant, bulk modulus, melting temperature, etc.) [52]. To correctly account for the high energy collisions that take place during implantation cascades, splines of empirical potentials to repulsive pair potentials are used [53]. Within CMD simulations the dynamics of atoms is obtained by the numerical integration of Newton's equations. These simulations showed that light ions generate dilute damage in the form of isolated FPs or small defect clusters (as observed in BCA calculations), while heavier ions can also generate larger defect clusters and amorphous regions [54-56]. They also showed that the energy deposition conditions in molecular implants result in the generation of craters [57-59], structures that cannot be predicted either by BCA. Regardless the detailed description of generated damage at the atomic level provided by CMD simulations, the associated high computational cost makes their use impractical for simulating a full implantation process (i.e. thousands of cascades on the same target with dynamic annealing during the intercascade time interval).

Different approaches have been made to develop simulation techniques and models that accurately predict the damage structures resulting from collision cascades with a relatively low computational cost $[55,60]$. One possibility consists on using CMD simulations to gain insight on the physics governing the damage generation mechanisms 
occurring during collision cascades [61-64], and then employing this information to develop advanced but simpler damage generation models $[65,66]$.

Whereas the atomic interactions occurring at the ballistic regime of energetic atoms (i.e. when their energy is well above $E_{d}$ ) can be considered as binary collisions with the closet target atom, multiple interactions with target atoms become important as the energy of moving atoms decreases. CMD simulations showed that energy transfers among atoms below $E_{d}$ can generate amorphous regions as the result of a competition between melting of the lattice and out-diffusion of the deposited energy [63]. This was also observed from ab-initio simulations [67]. To form an amorphous region by this mechanism, it is necessary that the deposited energy remains concentrated locally long enough for melting to occur before it out-diffuses. The quantification of CMD results allowed to develop a simpler model $[65,66]$ that defines the final number of displaced atoms $N_{D A}$ resulting from the evolution of $N$ initial moving atoms with an initial average energy $\rho$ (in $\mathrm{eV} /$ atom), as:

$$
N_{D A}=\frac{N \rho-E_{T}(N)}{D_{C}(N)}
$$

where $E_{T}(N)$ and $D_{C}(N)$ are the threshold energy density for damage production and the damage generation cost, respectively. According to Eq. 2, it is necessary first to overcome an energy density threshold, given by $E_{T}$, in order to reach an initial disordered state. To keep atoms permanently displaced, or at least a fraction of them, an additional amount of energy is required. $D_{C}$ controls the final number of displaced atoms that are generated with the remaining energy density. As $N$ increases, both $E_{T}(N)$ and $D_{C}(N)$ decrease, because it is easier to move an atom from its perfect lattice position and keep it displaced when its neighboring atoms are also energetically excited. The transition in damage generation mechanisms between ballistic (for $N=1$ in Eq. 2) and thermal spike regimes (for larger values of $N$ in Eq. 2) is naturally included within this dependence. The model also accounts for the nonlinear generation mechanisms that appear in energy spikes during molecular implants. In particular, this model was applied to simulate the implantation of single octodecaborane molecules into Si [58]. Figure 1 shows the damage predicted using the improved BCA model, together with the result from time-expensive CMD simulations. As it can be seen, the dimensions of the obtained disordered region applying the improved damage model are in good agreement with the results of CMD simulations, while the total calculation time was reduced in a factor of $10^{3}$ [66]. 

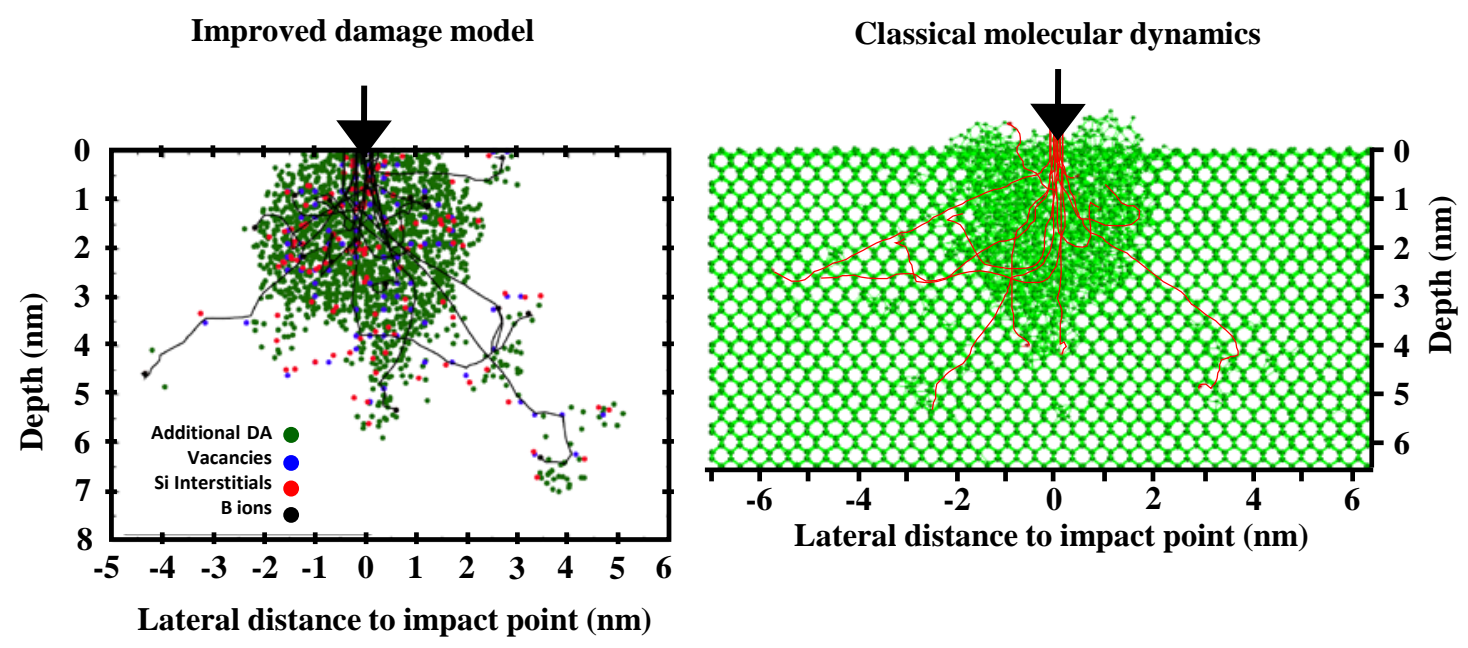

Fig. 1. Depth projection of damage generated by an octodecaborane molecule $\left(\mathrm{B}_{18}\right)$ cascade of $9 \mathrm{keV}$ (500 eV per B atom) obtained with the improved damage model (left) and from CMD simulations (right). A conventional BCA code only provides the position of FPs generated by the B ions. The improved damage model adds the disordered atoms (DAs) generated through multiple interactions, which constitute the main volume of the amorphous region and cannot be obtained only by the superposition of individual cascades (improved damage model results from Ref. [36], and CMD simulations from Ref. [58]).

This general framework can be applied to other materials with proper calibration. In particular, it was used to understand damage generation efficiency in Ge [68], since Ge has attracted much attention in the microelectronic industry as a complementary material to Si $[69,70]$ as well as in radiation detection applications [71,72]. This material is easily damaged by incident radiation and amorphizes at implant doses much lower than those required for $\mathrm{Si}$ [72-74]. CMD simulations indicate that the number of displaced atoms is always larger in Ge than in Si for equivalent deposited energy conditions [68]. In the case of $\mathrm{Ge}$, the melting mechanism dominates over energy out-diffusion due to a lower melting temperature and thermal conductivity than in Si. The comparative values of $E_{T}$ and $D_{C}$ for Si and Ge are shown in Fig. 2. $E_{T}$ is similar in both materials but $D_{C}$ is significantly lower in Ge than in Si. Thus, there is a higher efficiency of the thermal spike phase in Ge in comparison to $\mathrm{Si}$ which accounts for the enhancement of damage production in Ge compared to Si. 


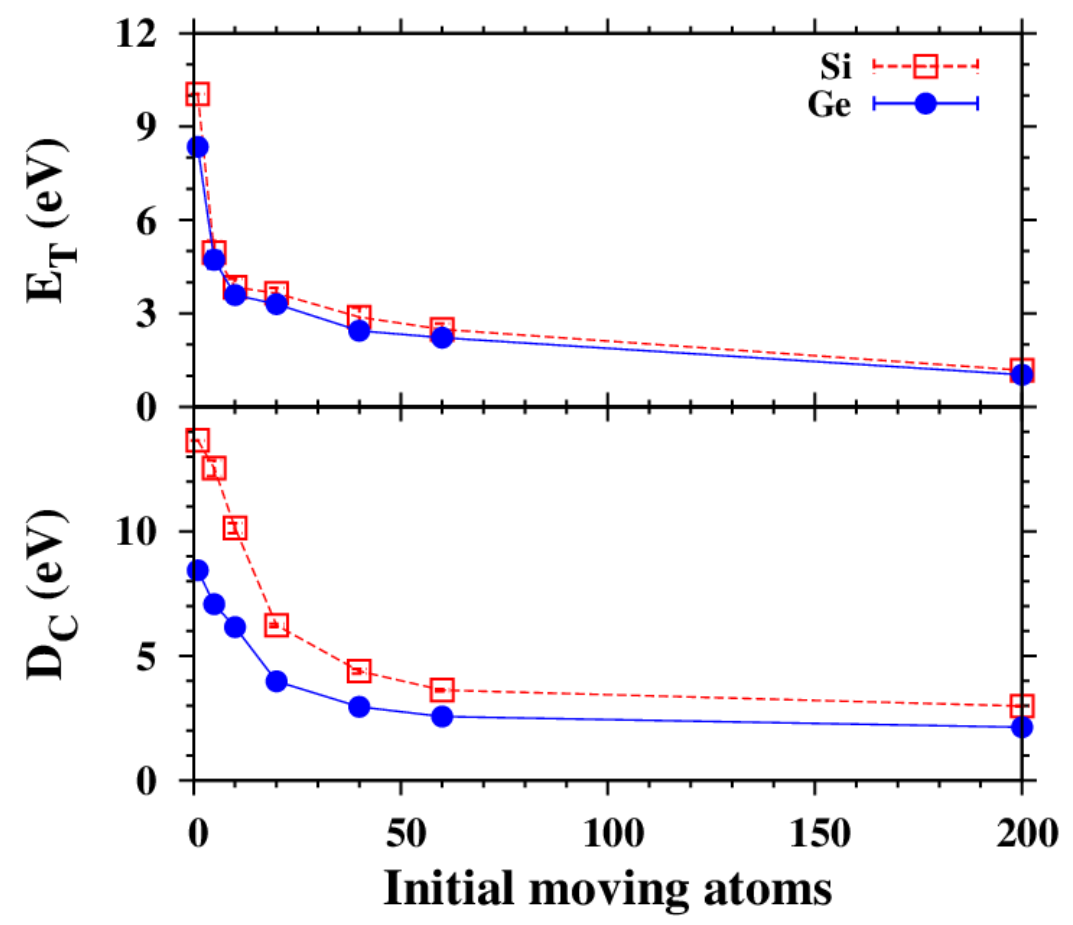

Fig. 2. Threshold energy $E_{T}$ and damage generation cost $D_{C}$ as a function of the number of initial moving atoms in Si and Ge (from Ref. [68]).

This framework has been also applied to understand how damage generation mechanisms can be affected by different factors, such as the presence of free surfaces and/or interfaces. It is important to quantify the role of surfaces because new device architectures (FinFETs, nanowires) have a high surface-to-volume ratio. Different experiments showed that the generation of damage near surfaces was enhanced [75-78] and some CMD simulations also suggested this behavior [79-82]. Experimental findings were initially explained considering that there might be a reduced amorphization threshold near surfaces. Other experiments showed that defects generated near the surface could have a higher stability [78]. Using CMD simulations we have quantified the role of surfaces in the generation of damage. Fig. 3 shows the evolution of $E_{T}$ and $D_{C}$ as a function of the distance to the (100) Si surface. Both $E_{T}$ and $D_{C}$ decrease with respect to bulk values when approaching the surface. If we consider the shallowest recoils, the energy required to permanently displace one atom $\left(E_{T}+D_{C}\right)$ is only $13.7 \pm 1.7 \mathrm{eV}$ for the (100) Si surface. This energy is approximately 38\% lower than the bulk value, which results in the enhancement of damage generation near the surface. 


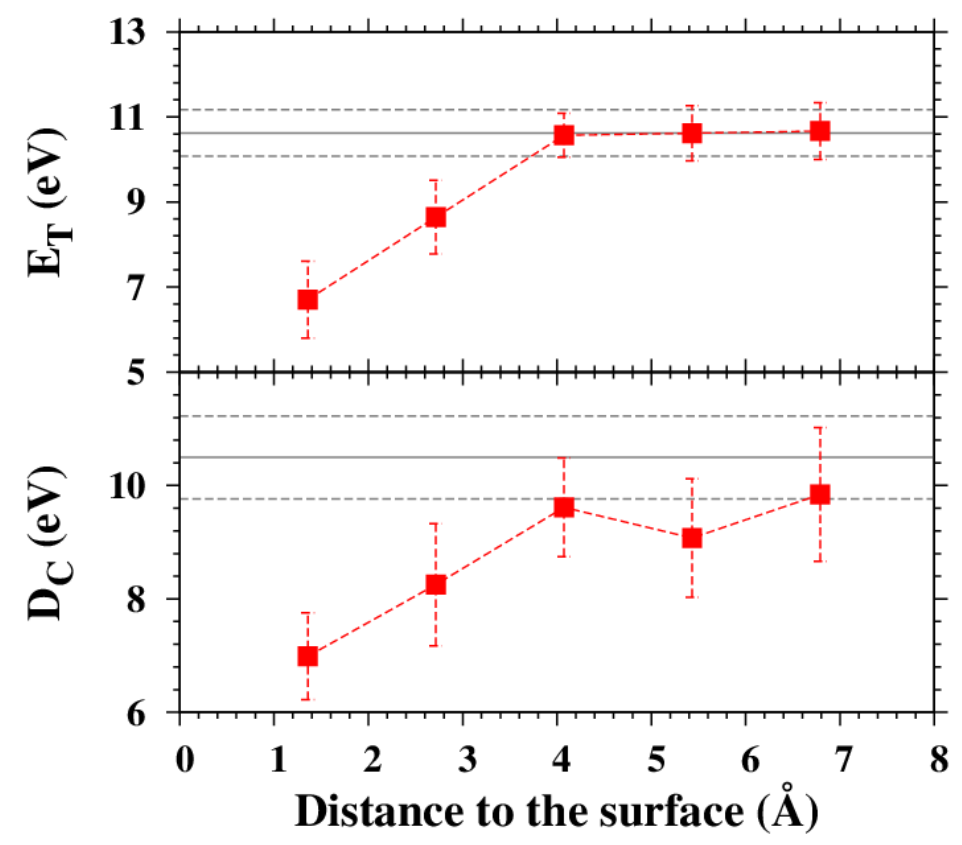

Fig. 3. Threshold energy $E_{T}$ and damage generation cost $D_{C}$ as a function of depth for the (100) Si surface. Horizontal solid lines show the bulk values, and horizontal dashed lines indicate their standard deviations.

\section{Modeling of amorphization and regrowth: from empirical models to detailed lattice kinetics}

Damage generated by implanted ions may accumulate leading eventually to the crystalline to amorphous transformation of the irradiated area. The dopant implantation process itself can be self-amorphizing, especially when heavy ions and/or high doses are used. But even for light ions, a preamorphizing implant is usually performed to create a continuous amorphous layer (a-layer) in which dopants are later introduced. The formation of the amorphous phase is beneficial as it limits ion channeling (which can distort the implant dopant profile) and during the subsequent solid phase epitaxial regrowth (SPER) step a high concentration of dopants can be incorporated into electrically active positions $[49,83]$. In addition, recrystallization takes place at relatively low temperatures [84], and the regrown layer has a low defect density because during SPER of a continuous a-layer excess Si self-interstitials are swept to the surface or buried interface, where they are annihilated [85]. Remaining damage, known as end-of-range (EOR) defects, is only a fraction of all generated defects during the implantation step, and is located beyond the initial a/c interface. The amount and location of EOR defects is 
determined by the thickness of the a-layer [55]. Therefore, it is essential to model with great accuracy the depth of the a-layer, as small variations may lead to large changes in the predicted residual damage.

During the ion implantation process there is a competition between damage generation and dynamic annealing, i.e. damage recovery that takes place as the implant proceeds. In fact, the intensity of the dynamic annealing during the implant may allow the accumulation of the generated damage or, on the contrary, the recombination of most of it, avoiding the creation of an amorphous region. Implant parameters such ion mass and energy, dose, dose rate and substrate temperature control the intensity and duration of dynamic annealing. By selecting the right implant parameters, dynamic annealing during the ion implantation process can be appropriately engineered to control damage accumulation. The optimization of the a-layer depth can significantly boost the efficiency of thermal treatments on the removal of EOR defects both in planar and 3D devices. If most of the irradiated region transforms into amorphous (by reducing the substrate temperature during implant, for example), most defects are swept to the surface during SPER and only a small fraction remains as residual damage [86]. In other cases, as it occurs when planar ultra-thin silicon-on-insulator (SOI) devices and FinFETs are fully amorphized [87], an incomplete or defective regrowth causes the degradation of device performance and variability [88], and implant parameters should be selected to enhance dynamic annealing and so avoid the formation of the amorphous phase.

The relevance of amorphization on semiconductor industry has motivated the development of several phenomenological and structural models to explain ion-beam induced amorphization and thermal recrystallization in Si [89-94]. These models, compatible with process simulators, must be able to predict the onset of amorphization and its dependence on implant parameters.

\section{Critical energy/defect density model}

The critical energy density model assumes that the implanted region turns amorphous when an energy density threshold (typically in the order of $5 \times 10^{23} \mathrm{eV} / \mathrm{cm}^{3}$ ) is exceeded [89,90,95], or a critical defect concentration (CDC) is reached (typically in the order of $1.15 \times 10^{22} \mathrm{~cm}^{-3}$ ) $[89,96]$. However, when the theoretical damage profile is compared with the experimentally measured position of the a/c interface, the CDC value required to obtain a good fitting is not unique, and variations of more than one order of 
magnitude have been reported [54]. The CDC value has been found to depend on implant parameters such as ion mass, dose rate and implant temperature, among others, which is associated to the effects of dynamic annealing on damage accumulation. Variations on the CDC value introduce uncertainty in the position of the a/c interface. In turn, as it is shown in Fig. 4, a small change in the position of the a/c interface may result in a large variation in the amount of residual damage remaining after SPER [97].

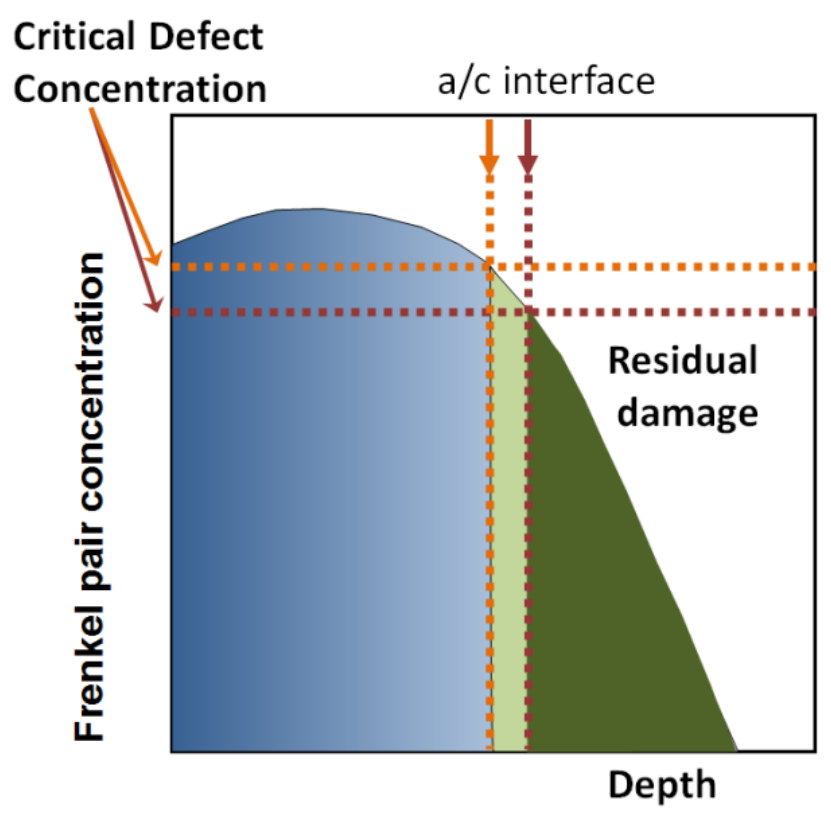

Fig. 4. Schematic that shows the a-layer depth and the amount of residual damage associated to two different CDC values.

\section{$\underline{\text { Atomistic KMC models }}$}

To take into account dynamic annealing during the implant, a key factor in modern ion implantation strategies, improved models have been developed that provide a detailed atomistic description of damage generation and accumulation during ion implantation. A unifying and consistent view of amorphization and recrystallization processes is provided by the non-lattice atomistic amorphization model based on the bond defect or IV pair [91]. The IV pair is a metastable defect structure generated by the incomplete recombination of an interstitial and a vacancy [98], or as a result of a pure ballistic process [99]. It consists of a local rearrangement of bonds in the crystal with no excess or deficit of atoms [100,101]. CMD simulations indicate that the IV pair as an individual defect is not stable enough to promote amorphization, but the stability of the IV pair increases with the presence of other IV pairs in the surroundings [29]. With the use of CMD simulations 
we have determined that the mean lifetime of IV pairs increases as approaching a free Si surface as represented in Fig. 5. This retardation of IV pair recombination near free surfaces causes the formation of twin defects in FinFET devices [87,102].

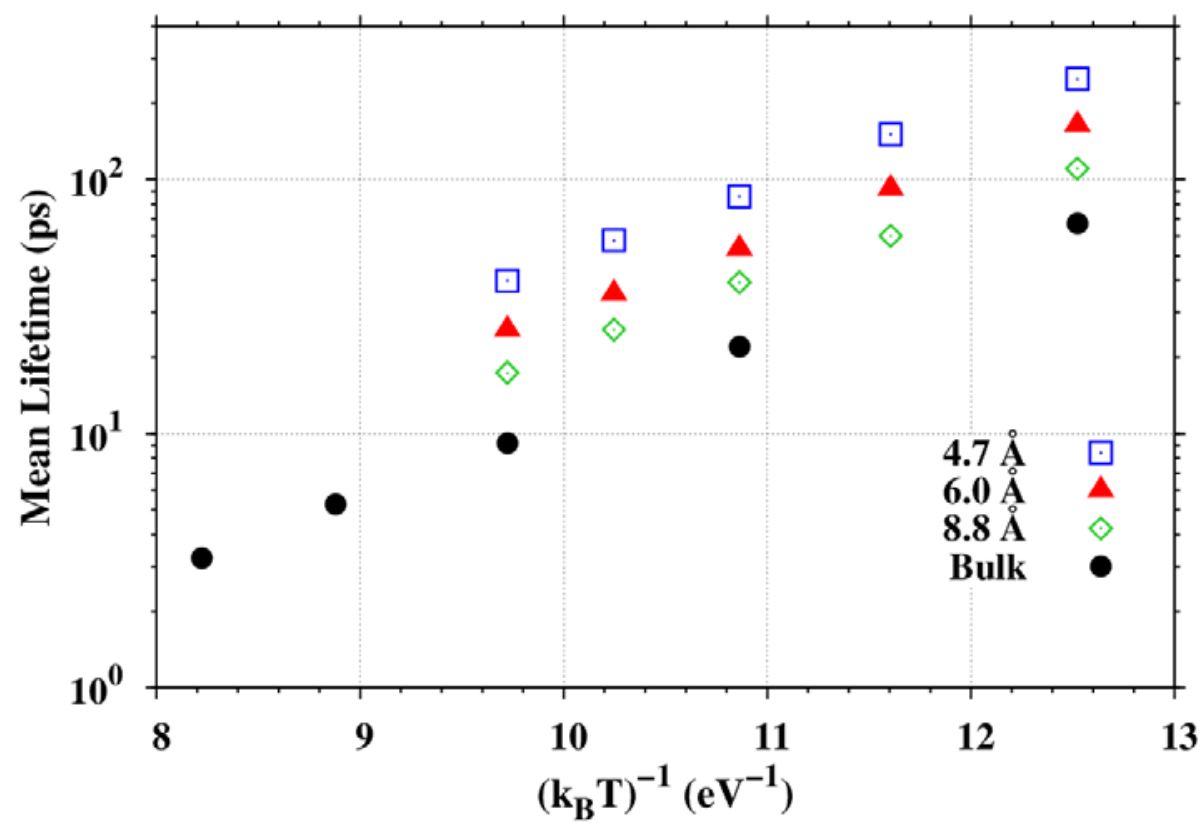

Fig. 5. Arrhenius plot of the mean lifetime of IV pairs as a function of the distance to the (100) Si surface. Results in the bulk are also shown for comparison.

In the KMC model proposed by Pelaz et al. [91] the IV pair is used as the elementary unit to describe the amorphous phase, and each IV pair is characterized by the number of neighboring IV pairs. IV pair recombination is modeled as a thermally activated process whose activation energy depends on the number of neighboring IV pairs. The local characterization of the disordered atoms allows the model to capture any damage morphology that may arise from irradiation cascades. Figure 6 is a schematic that shows a continuous a-layer and several amorphous pockets (a-pockets). Isolated IV pairs and those belonging to convex regions (like A and B in Fig. 6), have fewer neighboring IV pairs and recombine faster, shortening the lifetime of irregular a-pockets compared with more compact structures [103,104]. IV pairs at an a/c interface (C) have fewer neighboring IV pairs than those embedded within the amorphous region (F), and therefore recrystallization (IV pair recombination) will begin at the interface. The recombination of the first IV pair at the planar interface (C) starts a triggering mechanism in which its neighbors ( $\mathrm{D}$ and $\mathrm{E}$ ) are the most likely ones to be recombined next (since they have lost a neighbor), which leads to the complete recrystallization of the whole monolayer. 


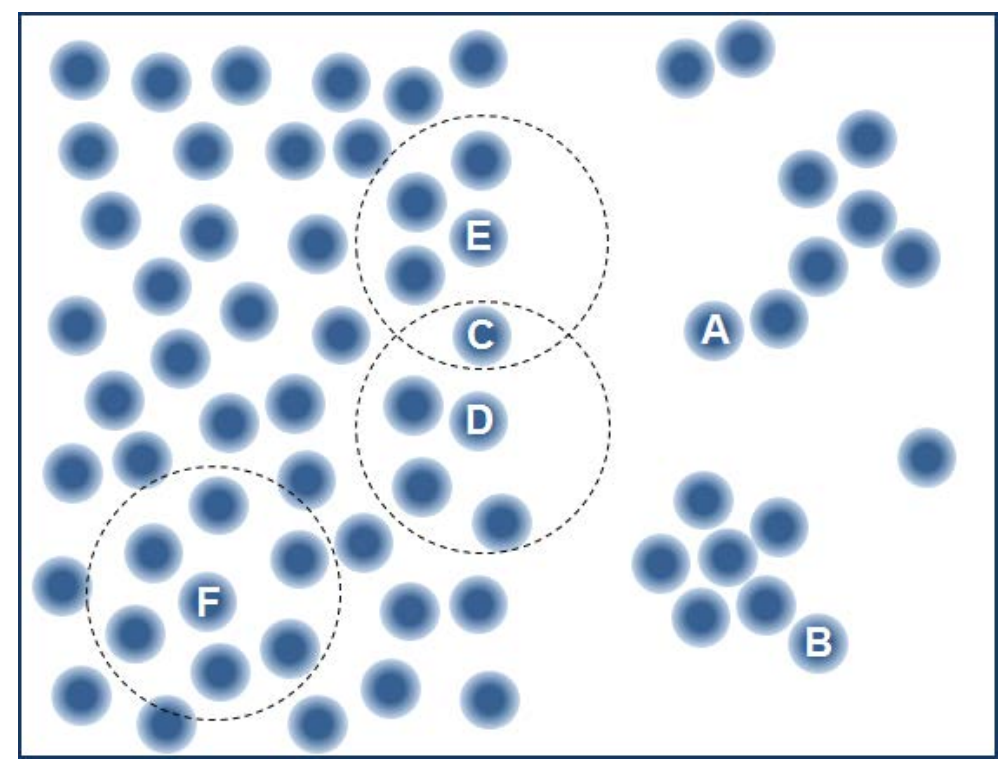

Fig. 6. Schematic of a damage distribution in which a continuous a-layer and several apockets are shown. IV pairs are represented by solid circles. For some IV pairs a dotted circle indicates their neighboring IV pairs.

To optimize CPU time and memory requirements, some KMC models implement a less detailed description of the amorphous phase, using the a-pocket as the basic amorphous structure [93]. A-pockets are considered as 3D irregular-shaped agglomerates of an arbitrary number of interstitials and vacancies and trapped impurities. Their recrystallization is a thermally activated process whose activation energy depends on the minimum number of interstitials and vacancies they contain, i.e. the overall number of IV pairs, without taking into account the a-pocket morphology [105]. The simulation cell is divided in small boxes that are considered to turn amorphous when the defect concentration associated to the a-pockets within the box exceeds the amorphization threshold. Recrystallization of each one of these boxes occurs as a whole at a rate given by the experimental regrowth velocity of a planar a/c interface.

\section{$\underline{\text { Lattice KMC models }}$}

Non-lattice KMC models cannot simulate the anisotropy of SPER with substrate orientation, being 20 times faster for $\{100\}$ interface orientation with respect to $\{111\}$ orientation [106]. SPER is also strongly dependent on stress [107]. Stresses can be externally generated or appear as a result of the $2 \%$ volume expansion of amorphous $\mathrm{Si}$ with respect to crystal Si [108]. CMD simulations have shown that the anisotropic regrowth of the amorphous phase in thin film layers results in twin defects [102,109,110], 
and that when significant strains are present, a slow-down in zones with high shear strain produces the evolution of ledges that collapse and evolve into Frank partial loops [111]. As mentioned previously, while CMD is very useful to get physical insight into the SPER process in confined regions, it is a very time-consuming technique that cannot be directly applied to the simulation of actual processing sizes and times.

To overcome the limitations of non-lattice KMC models in a computationally efficient code, Martin-Bragado et al. developed a lattice KMC model that reproduces different planar SPER velocities and the formation of defects during regrowth [94,111113]. Stress information can be extracted from finite-element methods [111] and fed back and forth to the lattice KMC code. The model is based on the assumption that each atom in the amorphous phase needs to form two undistorted bonds with the crystal to be incorporated to the crystalline phase [106]. As it is shown in Fig. 7, this happens for all atoms at $\{100\}$ oriented surfaces, but it requires the simultaneous recrystallization of two or three atoms for the $\{110\}$ and $\{111\}$ surfaces, respectively. Regrowth kinetics is modeled as a sequence of atomic recrystallization events, whose frequency is determined by a unique activation energy but with three different pre-exponential factors, characterizing the growth rates of $\{100\},\{110\}$ and $\{111\}$ orientations. Thus, the anisotropy on the regrowth rate for the three basic planar orientations during SPER is simulated as well as the formation of twins on $\{111\}$ planes, the slowed recrystallization near corners as a consequence of the shear strain, and the formation of dislocations where two recrystallization fronts meet $[94,111,114]$.

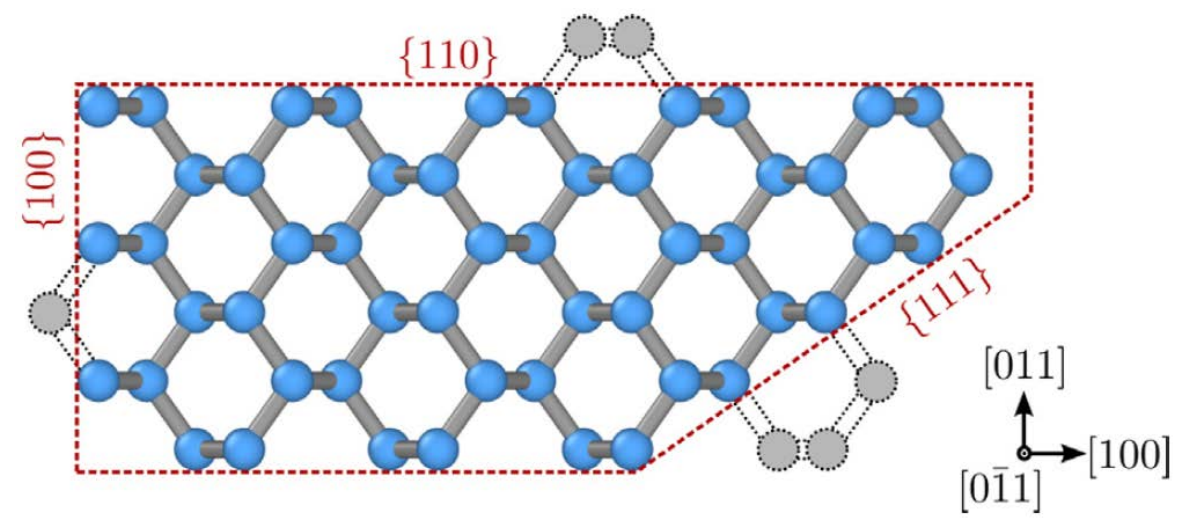

Fig. 7. Atomistic configurations for $\{100\},\{110\}$ and $\{111\}$ local recrystallizations. Si atoms in the crystalline phase are delimited by the dashed line. Sites to be occupied by atoms from the amorphous phase to induce a recrystallization event are indicated with dotted lines (from Ref. [113]). 


\section{$\underline{\text { CMD models }}$}

In the case of conventional planar devices, SPER produces the complete regrowth of the amorphous region up to the Si surface, with good crystalline quality and high dopant activation level in the regrown layer [109,115,116]. However, in the case of 3D nanometric multigate devices, SPER is not as straightforward as in bulk Si [87,117-119]. In fact, experiments show that in narrow planar ultra-thin SOI devices, FinFETs and nanowires, regrowth is imperfect, line defects starting at the lateral interfaces are formed in the implanted regions, and polycrystalline material is nucleated beyond $[87,120]$. These regrowth features, which are more acute in narrower structures, are serious concerns because of the degradation of device performance and variability [88,121].

CMD simulations have reproduced the observed experimental behavior regarding the defected recrystallization of FinFET structures in Si [102], as shown in Fig. 8. Duffy and coworkers fabricated sub-20nm wide Si fin structures on (100) SOI wafers, patterned in the $<110>$ direction [87]. After the implantation step, the fin body was fully amorphized and only the bottom portion remained crystalline (see Fig. 8(a)). During subsequent annealing, soon $\{111\}$ stacking faults (twins) started forming at the sides. Beyond them, polycrystalline Si was generated (see Fig. 8(b)). Thus, despite the presence of a lattice template at the bottom of the fin, SPER was highly defected, which produced an increase of the on-resistance of the final device [88,121]. On the contrary, in bulk Si systems, where regrowth occurs perpendicular instead of parallel to the interfaces, recrystallization is almost perfect [109]. Figures 8(c) and 8(d) show the initial and final snapshots taken in a CMD simulation of the annealing of a $10 \mathrm{~nm}$ wide Si fin surrounded by amorphous material (crystal orientations are the same as in the previously described experiment) [102]. SPER proceeds from the bottom crystal seed but at the lateral interfaces crystallization is hindered. Meanwhile, the recrystallization front in the center of the fin advances in the $<100>$ direction. The influence of the regrowth retardation due to the presence of lateral interfaces propagates diagonally, which makes the regrowth front to adopt the typical form of an arrow tip limited by $\{111\}$ planes. Spontaneous nucleation of crystal grains with random orientations occurs in the amorphous region beyond such stacking faults, which produces polycrystalline Si as observed in Fig. 8(d). 

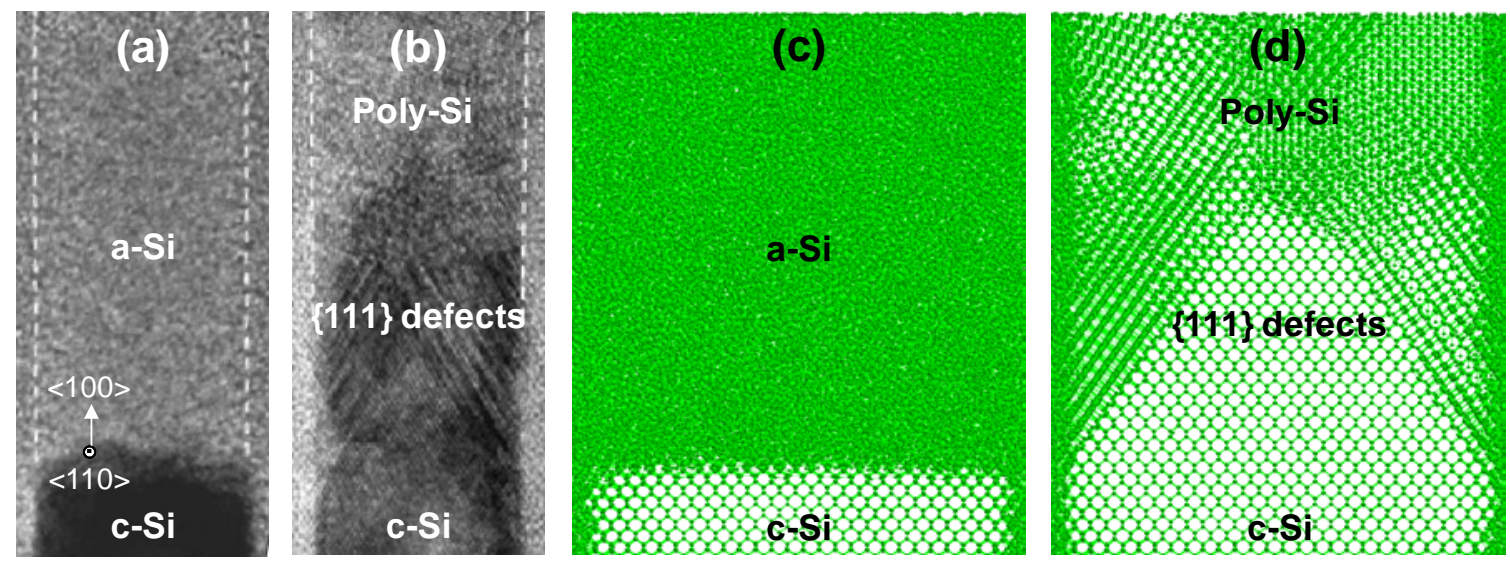

Fig. 8. Transmission electron microscopy images of a sub-20 nm wide Si fin after (a) the implantation and (b) the subsequent rapid thermal annealing (from Ref. [87]). (c) Initial and (d) final snapshots from the CMD simulation of the annealing of a $10 \mathrm{~nm}$ wide fin (from Ref. [102]).

CMD simulations show that defected regrowth is due, in the first place, to the slowing down of the recrystallization velocity in the vicinity of the a/c interfaces. This is basically due to the absence of a crystal template beyond such interfaces [102,122]. In the particular orientation of the fin body considered in the experiment by Duffy et al. [87], recrystallization fronts, retarded at the lateral interfaces but continuing in the fin body center, catch up with $\{111\}$ planes. Regrowth along the $<111>$ direction is slowest and shows frequent defects in the stacking sequence [106,123]. Due to the consequent recrystallization slow down, spontaneous nucleation of randomly oriented crystal grains in the amorphous region beyond the staking faults becomes the fastest regrowth mechanism, producing the formation of polycrystalline Si [124]. In planar devices, recrystallization is not hindered and crystal grains have not enough time for nucleating before full regrowth occurs.

CMD simulation results can be used to suggest alternatives to improve SPER in multigate devices. One possibility consists of changing the fin orientation. Fig. 9 shows the initial and final snapshots taken from the CMD simulation of the annealing of a 10 $\mathrm{nm}$ wide Si fin, now aligned along the $<100>$ direction [102]. Recrystallization is improved with respect to the $<110>$ oriented fin. This is because the influence of the lateral interface now propagates diagonally along $\{110\}$ planes. SPER along the $<110>$ direction is faster that in $<111>$ and does not show defects in the stacking sequence so frequently [106,123]. 

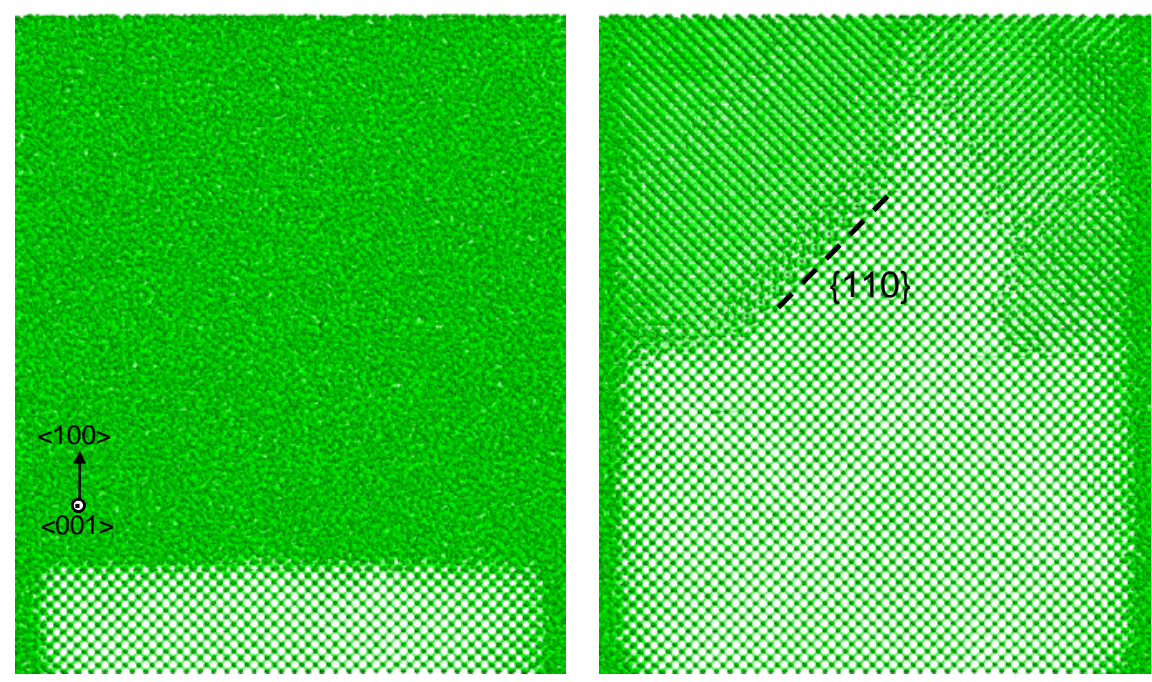

Fig. 9. Initial and final snapshots taken from the CMD annealing of a $10 \mathrm{~nm}$ wide fin structure aligned along the $<100>$ direction (from Ref. [102]).

Another alternative to improve SPER consists of leaving a crystalline seed in the middle of the fin (by selecting appropriate implant parameters in the experiment). Figure 10 shows the initial and final snapshots taken from the CMD simulation of the annealing of a $10 \mathrm{~nm}$ wide $\mathrm{Si}$ fin aligned along the $<110>$ direction, with a thin crystalline seed (just $1 \mathrm{~nm}$ thick) in the middle of the fin body. In this case there are two recrystallization fronts, one advancing parallel to the lateral interfaces in the $<100>$ direction and another one perpendicular to lateral interfaces in the $<110>$ direction. Even though regrowth is again limited by $\{111\}$ planes, recrystallization quality is significantly improved with respect to CMD simulations shown in Fig. 8. SPER is good even in the case that the thin crystalline seed in the middle of the fin is highly damaged (as it would be expected after the implantation step) [102].

SPER in nanowires is even more challenging than in the case of FinFET structures: while in the latter case the crystal phase is confined within the amorphous material in just one dimension, in the former case of nanowires it is confined in two. This fact hinders the correct SPER of the surrounding amorphous phase as several recrystallization fronts with different associated velocities interact and merge. CMD simulations show that SPER of nanowires renders more defective crystalline material when compared with the regrowth of fin structures with the same dimensions and orientations [102]. These CMD results are in striking good agreement with the in-situ experimental observation of the regrowth of nanowires [120]. 

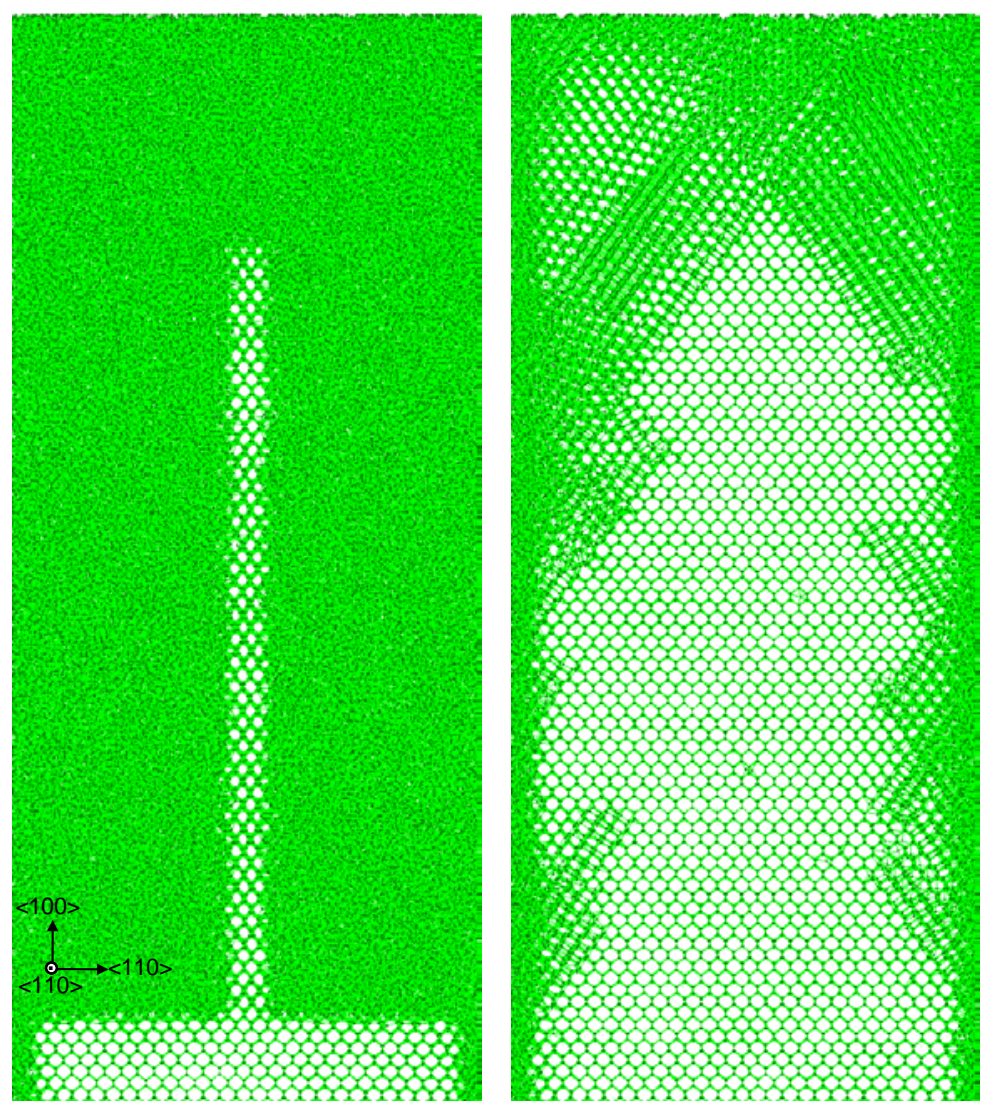

Fig. 10. Initial and final snapshots taken from a CMD annealing of a $10 \mathrm{~nm}$ wide fin structure aligned along the $<110>$ direction with a $1 \mathrm{~nm}$ crystalline seed in the middle of the body (from Ref. [102]).

\section{Damage engineering by implant optimization}

The control of dynamic annealing during ion implantation is a promising strategy to minimize the concentration of EOR defects and facilitate their removal, or to prevent amorphization. This is especially challenging for FinFET devices due to the small volume involved in narrow fins, in which keeping the substrate crystallinity and a low defect density are critical issues. Damage engineering by the optimization of implant parameters requires a deep understanding of the physical mechanisms underlying damage accumulation and phase transformations, and especially of the role of implant conditions on dynamic annealing [54,92].

Implant energy determines the projected range of the implanted ions, i.e. the region where most damage is generated and amorphization begins [125]. Because of the enhanced stability of large a-pockets compared to dilute damage, damage accumulation 
is superlinear versus dose [126]. Once amorphization is reached, new cascades inside the amorphous region just move atoms already displaced from their lattice positions. More damage is added only when new cascades reach not-fully amorphous regions close to the continuous a-layer, which causes its widening. In self-amorphizing doping implants, dose and ion energy are selected to obtain the desired a-layer depth, or even several implants with different energies and doses are combined to ensure the formation of a continuous a-layer that extends to the surface, so that the excess defects contained inside the a-layer are swept towards the surface during SPER.

To accurately reproduce the onset of amorphization and the evolution of the alayer thickness with dose, models should account for defect accumulation and the progressive reduction of channeling as the implanted region turns amorphous. BCA codes that consider the target material as crystalline [37] reproduce channeling during the implant and the formation of damage tails, while those that consider amorphous targets result in more abrupt damage profiles [53]. However, as damage accumulates, the new incoming ions see a target that is gradually transforming from crystalline to amorphous. To take into account such effect, advanced BCA simulators include the feedback of accumulated damage for the next cascades [40,42,127-130].

Figure 11 shows KMC simulation results of the evolution of the a-layer thickness with dose [131], compared with experimental data from Maszara et al. [132], for $150 \mathrm{keV}$ Si implants at $82 \mathrm{~K}$. There is an initial fast increase of the a/c interface depth with dose until a given depth is reached, and then, the increase is very slow. Since at this temperature dynamic annealing is negligible and most damage is stable, the observed saturation is caused by the spatial distribution of generated damage. A schematic of the evolution of the damage profile with dose is plotted in Fig. 12, in which for simplicity the substrate is considered to turn amorphous when damage exceeds a threshold value. For low doses the damage profile is similar to that in a crystalline substrate, showing the channeling tail with a smaller slope. As dose increases, more damage is added and a wider a-layer is formed. As a consequence of damage accumulation, channeling is progressively reduced, giving rise to a steeper profile. Once the damage profile is very abrupt, a dose increase scarcely modifies the position of the a/c interface. These results show that increasing the implant dose may result in a more abrupt a/c interface, but it is not an efficient way to widen the a-layer. 


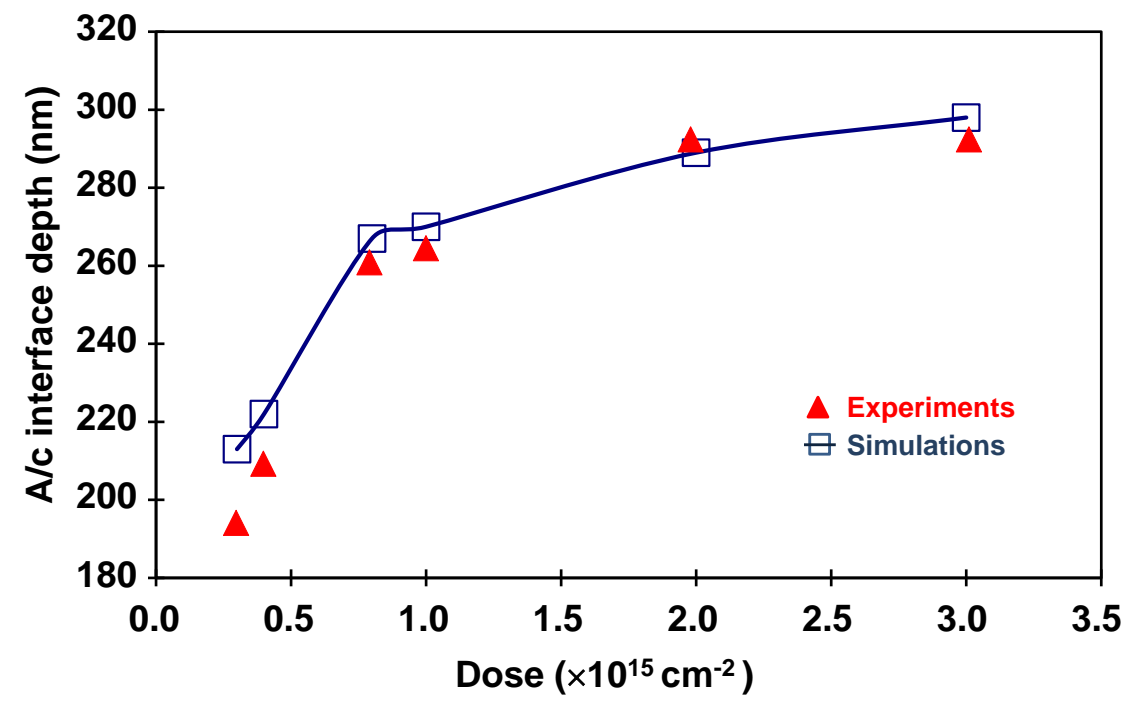

Fig. 11. A/c interface depth as a function of dose for a Si $150 \mathrm{keV} 1.56 \times 10^{12} \mathrm{~cm}^{-2} \mathrm{~s}^{-1}$ implant performed at $82 \mathrm{~K}$. Solid symbols represent experimental data extracted from Ref. [132]. Open symbols and the line correspond to simulation results (from Ref. [131]).

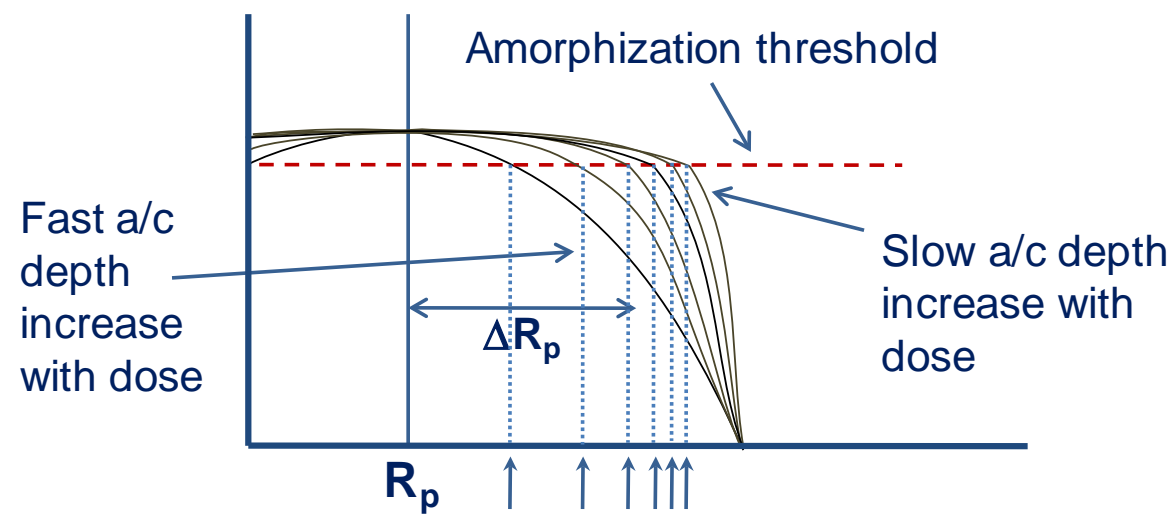

Fig. 12. Schematic of the evolution of the damage profile with dose. $R_{p}$ and $\Delta R_{p}$ represent the projected range and the lateral straggling of the implant, respectively. The dashed line shows the amorphization threshold, above which the substrate turns amorphous. The position of the a/c interface is indicated by small arrows.

The critical dose required for amorphization depends on ion mass. As the ion mass increases amorphization is possible with lower doses. While amorphization in Si can be easily achieved with $\mathrm{Sb}$ at doses in the order of $10^{14} \mathrm{~cm}^{-2}$, doses of at least $10^{16} \mathrm{~cm}^{-2}$ are needed to form a continuous a-layer with B ions at room temperature [133]. Differences do not only come from the amount of generated defects, but mostly because the 
morphology of damage strongly influences its dynamic anneal. The improved BCA model developed by Santos et al. (see Section 2) reproduces the amount and morphology of generated damage at atomic level by encompassing both the ballistic and the thermal damage generation regimes, characteristic of light and heavy ions, respectively $[65,66]$. The influence of morphology on damage stability is naturally captured by the amorphization model based on the IV pair, due to the local characterization of the disordered atoms (as shown in Fig. 6).

To illustrate the role of ion mass on dynamic anneal we show in Fig. 13 the damage evolution at room temperature for 100 cascades of low energy B and As implants from KMC simulations in which amorphization is envisioned as the accumulation of IV pairs [104]. While most generated damage by the B implant has not survived dynamic annealing, the larger mass of As results in larger and denser cascades which increase damage resistance to anneal (IV pairs are more stable as they have more neighboring IV pairs). For preamorphizing implants, the use of heavy ions such as Ge or Xe allows to form deeper a-layers with lower doses. N-type dopants (As) are usually self-amorphizing, while for p-type doping (B) molecular ions, such as $\mathrm{BF}_{2}$ or octodecaborane, can be used instead of monatomic B to promote self-amorphization and at the same time to achieve higher dose rates improving the throughput of implant tools [134,135].
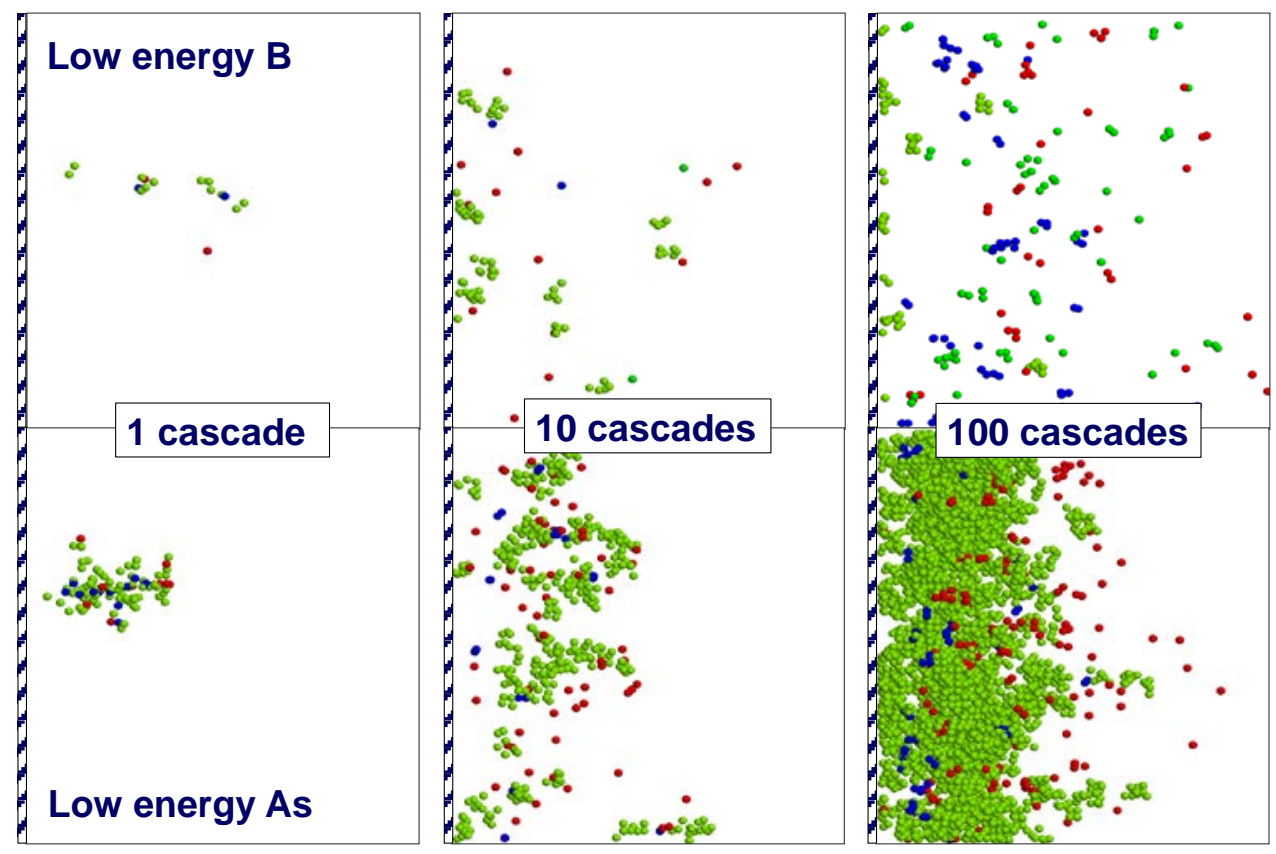

Fig. 13. Damage accumulation during 100 cascades of low energy B and As implants at room temperature from KMC simulations. IV pairs are shown as green dots, while Si interstitials and vacancies correspond to red and blue dots, respectively. 
The dose rate also affects the accumulation of damage by modifying the time of the dynamic anneal. This parameter specifies the temporal separation of implant cascades. If the dose rate is low, generated damage may have enough time to anneal out before the next cascade arrives into the same region. When the dose rate is high, damage generated by a cascade may overlap with damage from previous cascades favoring the formation of more stable defect structures which can accumulate [89,136]. We show in Fig. 14 experimental and KMC simulation results including the a-layer depth and the amount of residual damage after regrowth, for different dose rates [131]. As it can be seen, as the dose rate increases a wider a-layer is formed and the amount of residual damage is reduced. It must be noted that different implant architectures in commercial implanters may exhibit disparities in ion beam parameters which could affect process results $[137,138]$. Advanced KMC models take into account the specific time-structure of the beam of implant tools, since assuming an average dose rate does not capture all of the physics and may lead to incorrect results [139].

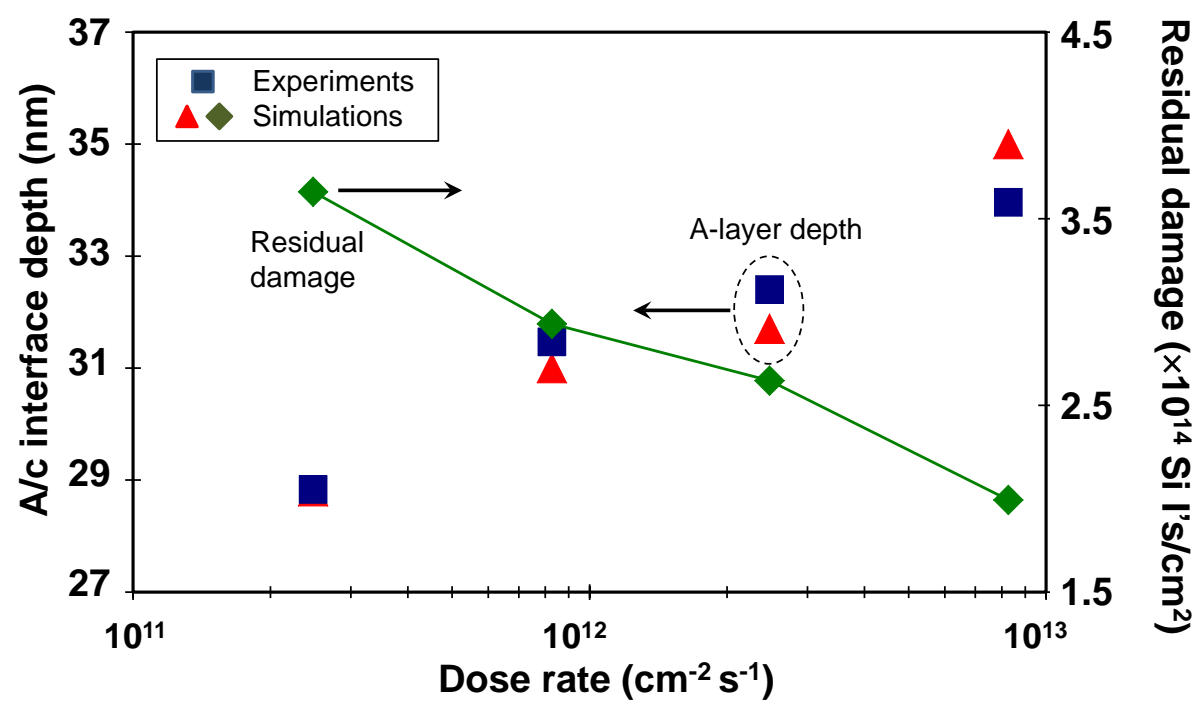

Fig. 14. Influence of the dose rate on the a/c interface depth and residual damage. $8 \times 10^{14}$ $\mathrm{cm}^{-2} 12 \mathrm{keV}$ Si was implanted at $293 \mathrm{~K}$ at dose rates ranging from $2.48 \times 10^{11}$ to $8.27 \times 10^{12}$ $\mathrm{cm}^{-2} \mathrm{~s}^{-1}$. Squares and triangles represent experimental (from Ref. [140]) and simulated a/c interface depths, respectively. Residual damage obtained in simulations is plotted as diamonds.

Wafer temperature may significantly rise during the implant process, especially for high doses and dose rates, if heat is not appropriately dissipated [141]. Precise control of wafer temperature during the implant is essential to limit device variability and it is 
also one of the main damage engineering strategies used nowadays [142-145]. Low implant temperatures are used to reduce EOR damage through the formation of a thicker a-layer that is cured during SPER. In this sense, cryogenic implants $\left(-100^{\circ} \mathrm{C}\right)$ have been proved to reduce sheet resistance and threshold voltage variability [142-144].

The problems associated to the imperfect regrowth of fully-amorphized thin films $[87,102]$ can be avoided by rising the substrate temperature during the implant, so that amorphization is prevented and the crystal integrity of the wafer is maintained. The socalled heated, raised or hot ion implantation is performed at an elevated temperature to enhance dynamic annealing avoiding damage accumulation. Hot ion implantation has been widely studied in the past [146] and has been applied for the source/drain (S/D) extension formation of Si-channel CMOS FinFETs [147-149]. Recently, hot ion implantation has been proved to enhance the performance of SOI CMOS FinFETs, resulting in better on/off characteristics and lower threshold voltage variability [150].

For a given implant condition there is a critical temperature above which amorphization is avoided. The critical temperature depends mainly on the ion mass and the dose rate [151,152], as it is shown in our KMC simulation results of Si and Ge implants reported in Fig. 15. Higher temperatures are required to prevent amorphization for Ge implants due to its larger mass.

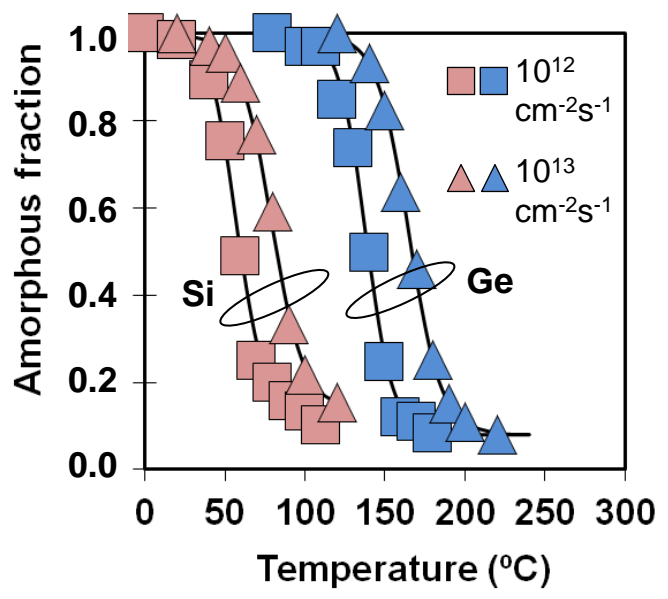

Fig. 15. Simulated amorphous fraction versus implant temperature and dose rate. Amorphization is prevented above a given critical temperature value.

KMC simulations can be used to predict the depth of the a-layer resulting from hot implants as a function of temperature, and the amount of residual damage remaining after recrystallization [153]. Results plotted in Fig. 16 indicate that a large amount of 
excess Si interstitials remains in EOR defects as the a/c interface depth is reduced. For temperatures high enough to prevent amorphization, intense dynamic anneal during implant can reduce the number of residual Si self-interstitials, but a larger number of selfinterstitial hops at high temperatures (see Fig. 17), mostly during implant, implies significant dopant diffusion [154].

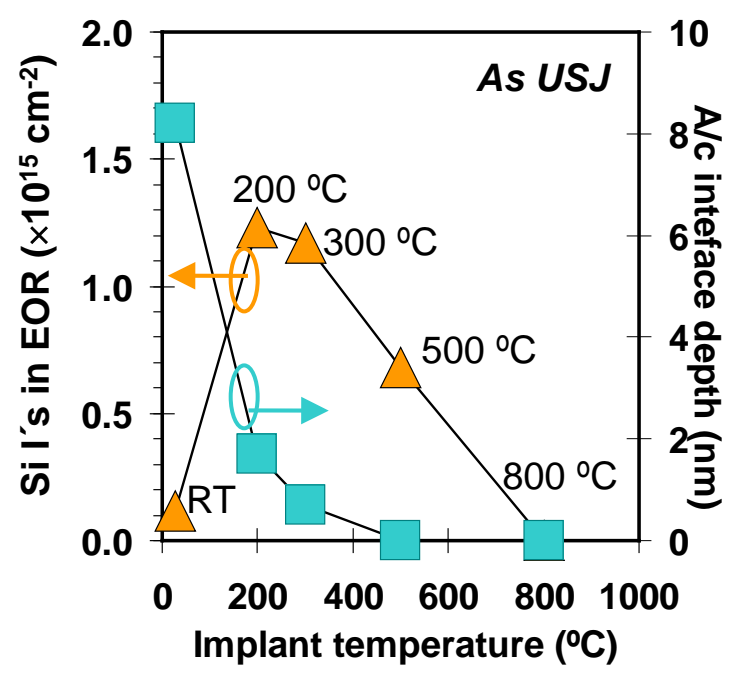

Fig. 16. Simulated a/c interface depth and EOR damage versus implant temperature for $5 \mathrm{keV} 10^{15} \mathrm{~cm}^{-2}$ As implants. A reduced a/c interface depth may result in more residual defects (from Ref. [153]).

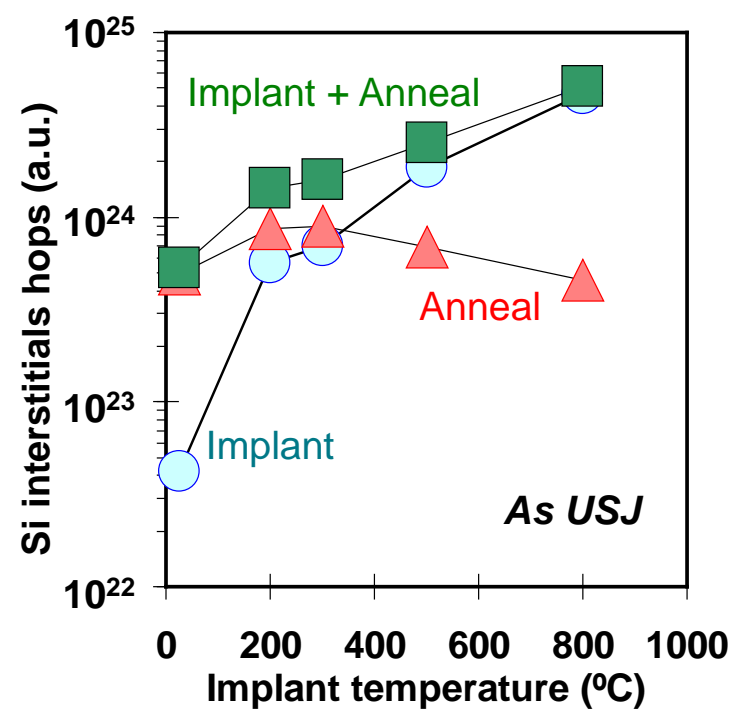

Fig. 17. Simulated Si interstitial hops versus implant temperature, during $5 \mathrm{keV} 10^{15} \mathrm{~cm}^{-2}$ As implants and rapid thermal annealing at $1050^{\circ} \mathrm{C}$. More interstitial hops mean more diffusion (from Ref. [153]). 


\section{Doping issues in FinFET devices: a challenge in 3D}

The development of 3D device architectures such as FinFETs has supposed a challenge for doping processes because high dopant concentrations along with uniform and conformal doping conditions are required. This has also increased the complexity of the characterization techniques used to assess the suitability of the created dopant profiles [23]. Thus, computer simulations become a good alternative to evaluate dopant incorporation, distribution and electrical activation in such devices. The particular geometry along with the reduced dimensions make atomistic simulations very appropriate to address doping requirements on FinFETs. In this section we illustrate how detailed models are able to provide insight into the issues associated to these devices.

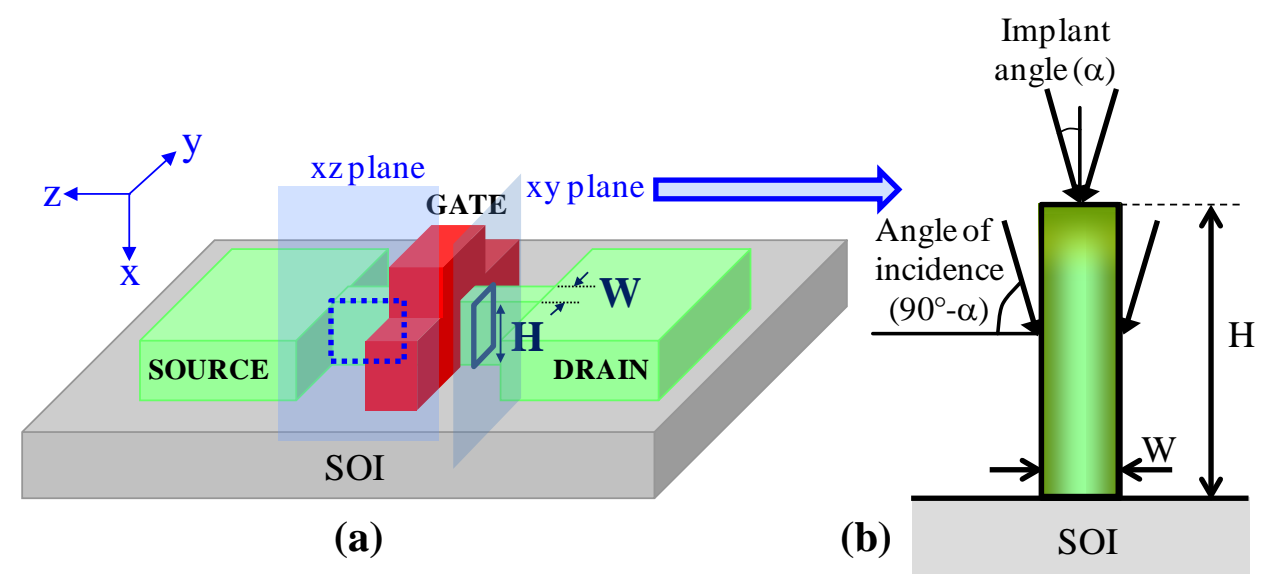

Fig. 18. (a) Schematic of a FinFET device with fin dimensions $H$ (height) and $W$ (width), where $x y$ and $x z$ planes are indicated to clarify cross sectional views that will be considered during this section. (b) Schematic of the dual quadrant implant procedure to incorporate dopants thorough the fin.

A schematic of a FinFET device is shown in Fig. 18. To achieve a good control of short channel effects (SCE) and off-state leakage currents, the fin width of FinFET devices must be narrow $[155,156]$, but the parasitic S/D resistance increases as the fin width is scaled down [88,157]. This challenge to optimize FinFET performance is complicated by the difficulties to dope the fin due to its vertical structure. Classical standard doping techniques like ion implantation may not be suitable for FinFET devices because of the 3D geometry. Several solutions to incorporate dopants in the fin are being explored such as tilted ion implantation [23,153,158], plasma doping [159-162] or vapor phase doping [163]. Among them, tilted ion implantation remains a strong candidate to introduce dopants into the fin, as it is a conventional and well established technique, 
although it suffers from specific issues that arise from the particular geometry of these devices. Figure 18(b) shows a schematic of the dual quadrant implant procedure typically used to dope the S/D regions, i.e. implantation from the left side and from the right side of the fin structure. Alternatively, plasma doping has gained interest due to the higher levels of conformality of fin doping and the lower levels of damage in the fin that can be achieved when compared to ion implantation, although it is also quite challenging due to secondary effects (sputtering/erosion, traps generation, etc.) that need to be addressed $[19,161,162]$.

\section{Dopant incorporation efficiency}

One of the main challenges is the efficient dopant incorporation into these vertical structures, as it directly affects the parasitic resistance $R_{S D}$ that defines the dynamic performance of FinFETs (high $R_{S D}$ values may limit the maximum drive current) $[158,164]$. This resistance is controlled by the geometry of the fin and the carrier concentration (determined by electrically active dopants) in the fin. As fins must be narrow in order to control SCE, reduction of fin resistance in the S/D region becomes critical, thus high-dose implants into the sidewalls of the fin are required. For ion implanted extensions the issue is greatly aggravated by the small tilt implant angle $\alpha$ required to avoid resist shadowing with dense fin pitches. The fin height-to-spacing ratio and the resist height determine the maximum implant angle so that the ion beam hits the foot of each fin, as illustrated in Fig. 19(a). Note that the tilt implant angle for the top surface implant is $\alpha$ whereas the angle of incidence to the sidewall surface is $90^{\circ}-\alpha$.

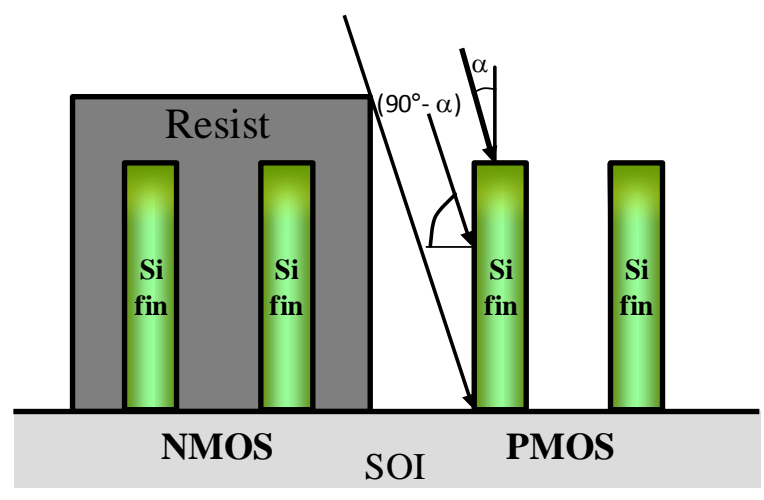

(a)

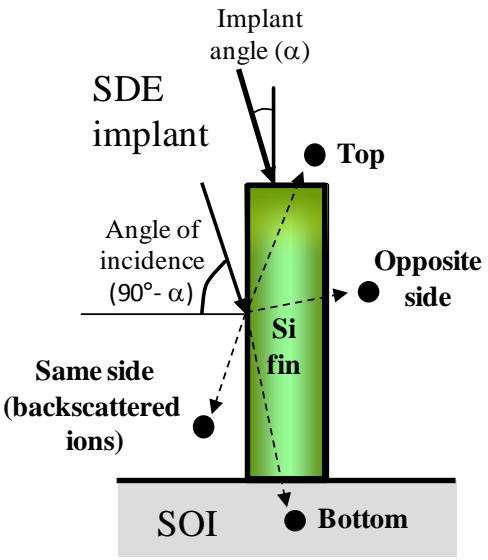

(b)

Fig. 19. (a) Schematic of the implant in dense fin array structures to illustrate the angle limitation. (b) Schematic of the different mechanisms of ion loss in a narrow fin structure. 
Simulations allow the identification and quantification of the different sources of dopant loss during ion implantation. Using the BCA code MARLOWE [37], integrated in the atomistic KMC simulator DADOS [27], it is possible to evaluate the dopant incorporation efficiency in the fin under different conditions of tilt implant angle $\alpha$, ion mass and implant energy. This simulation scheme also offers the advantage that the 3D nature of FinFETs is easily reproduced. As a first approximation to study dopant incorporation issues, dopant retention and loss were evaluated for implantation only into a lateral sidewall [165]. The schematic of the different mechanisms of dopant loss for sidewall implantation are shown in Fig. 19(b), which include backscattering during ion implantation (same side) and ion trajectories escaping from fin dimensions (opposite side, top and bottom). Figure 20 plots the percentage of ions retained in the fin and the percentage of dopant loss through all the different mechanisms shown in Fig. 19(b) for B and As implants, $10^{\circ}$ and $45^{\circ}$ tilted, into $10 \mathrm{~nm}$ wide fins at different implant energies. As it can be observed, main contributions to dose loss are backscattered ions (same side) and ion trajectories larger than the narrow fin width (opposite side). Simulations indicate that dose loss strongly increases as implant tilt angle decreases, mostly due to the increase of the amount of ions that are backscattered. Thus, for $10^{\circ}$ implants ( $80^{\circ}$ implants to the sidewall) only a small fraction of ions are incorporated into the fin mostly due to strong dose loss through backscattered ions at highly oblique angle of incidence to the sidewall. At $45^{\circ}$, the amount of retained dose is significantly higher than for $10^{\circ}$ implants as backscattering is greatly reduced. However, in narrow fins, a significant fraction of ions could also be lost because ion trajectories may escape from the fin, being this loss more significant in the opposite sidewall. This effect increases as the implant energy does, and it is more relevant for high tilt implant angles (lower incident angles to the sidewall). Finally, for a given implant angle and energy, heavier ions achieve higher levels of retained dopants since heavier ions penetrate deeper in the sample (less backscattering). A similar study could be done for top implantation although the loss of dopants is less significant as backscattering is minimized due to the small incident implant angles required to avoid shadowing.

Figure 21(a) summarizes the dependence of dopant retention on implant conditions, as predicted by BCA calculations [153]. The incorporation of dopants in the sidewall of the fin improves as tilt implant angle increases (due to the reduction of backscattering), but high implant angles are not allowed in dense fin arrays. For low tilt 
angles, medium energy implants achieve better levels of dopant incorporation. However, as the tilt implant angle increases, the use of medium implant energies results in dopant loss due to ions that escape from fin in narrow structures. Finally, heavier ions are generally better for dose retention. The inefficient dopant incorporation can be compensated with an increase in the implant dose. However, erosion caused by sputtering effects has to be taken into account as well. The simulated sputtering yield (target atoms extracted from a perfect Si surface per implanted ion) versus implant angle for B and As implants is plotted in Fig. 21(b). Sputtering (and therefore target erosion) is quite significant for small implant angles and heavy ions, while for light ions the effect is weak.
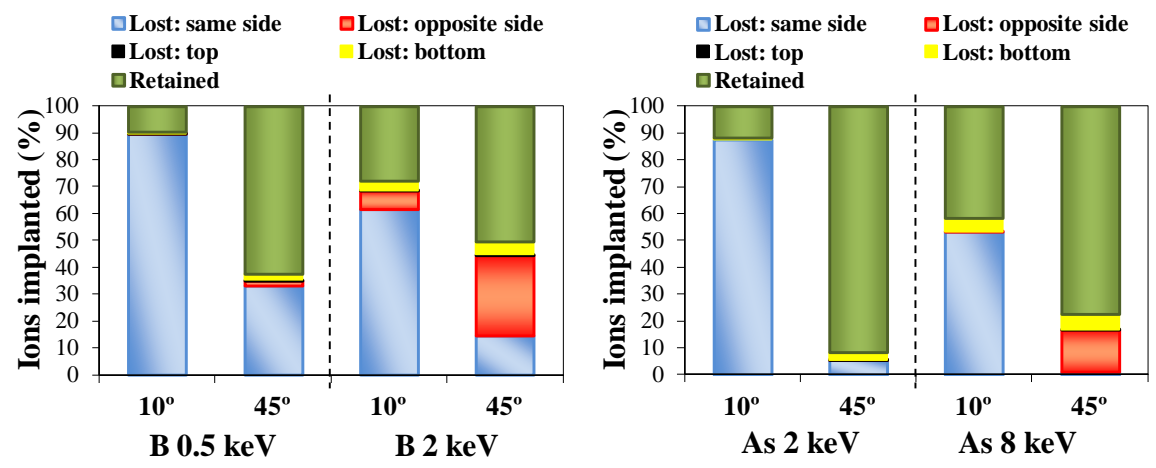

Fig. 20. Percentage of ions implanted and quantification of the different sources of dopant loss for B implants (left) and As implants (right) into $10 \mathrm{~nm}$ wide fins at tilt implant angles of $10^{\circ}$ and $45^{\circ}$ (angles of incidence to the sidewall of the fin of 80 and $45^{\circ}$, respectively) for low and medium implant energies (from Ref. [165]).
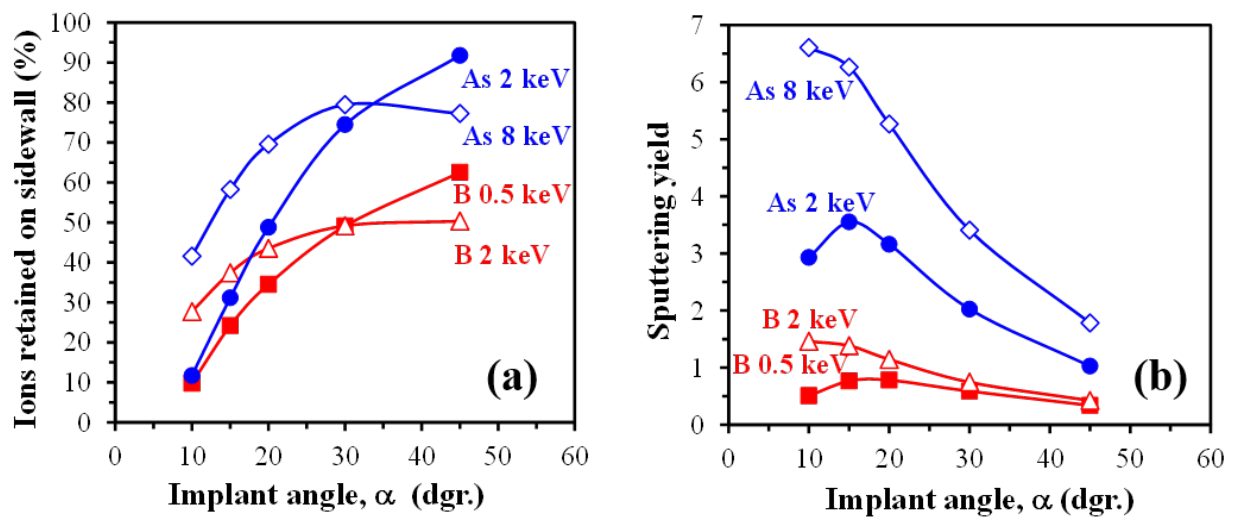

Fig. 21. (a) Simulated B and As sidewall dose retention versus angle in a $10 \mathrm{~nm}$ wide fin for low and medium energy implants. Small angle variations cause large changes in retention for small angles. (b) Sputtering yield versus implant angle for low and medium energy B and As implants. High energy heavy ions cause significant sputtering when they hit with a small angle (highly oblique to the sidewall) (from Ref. [153]). 
The experimental verification of the inefficient incorporation of dopants into the sidewalls for high incident angles and its dependence on implanted ion mass was later confirmed by secondary ion mass spectroscopy (SIMS) analysis performed in blanket wafers combined with SRIM code simulations reported by Duffy et al. [166]. These authors also demonstrated by device electrical simulations that both drive current and SCE control are very sensitive to sidewall dopant concentration. SIMS measurements [153] and Atom Probe Tomography (APT) data [167] for ion implanted fin structures also revealed more efficient dopant incorporation in the top compared to sidewalls due to complementary incident angles (see Fig. 18(b)), which complicates doping conformality as discussed in the next sub-section. As an example, Fig. 22(a) shows B concentration as a function of depth from a SIMS analysis of a $200 \mathrm{~nm}$ tall fin array implanted with $\mathrm{BF}_{2}$ at different tilt angles $\left(10^{\circ}\right.$ and $\left.45^{\circ}\right)$ and energies (medium and low) to a dose of $8 \times 10^{14} \mathrm{~cm}^{-2}$. The first dopant concentration peak is related to the top surface of the fin, whereas the plateau in the SIMS profile (around 30-200 nm) corresponds to the total sidewall concentration (i.e. both sidewalls of the fin). These experiments corroborate the inefficient dopant incorporation for low-energy low-tilt implants. In addition, the loss of dopants when using higher implant energies for $45^{\circ}$ tilted implants in narrow fins has been experimentally confirmed by the increase in the resistance of the fins as implant energy is increased [88,153], as it is shown in Fig. 22(b).
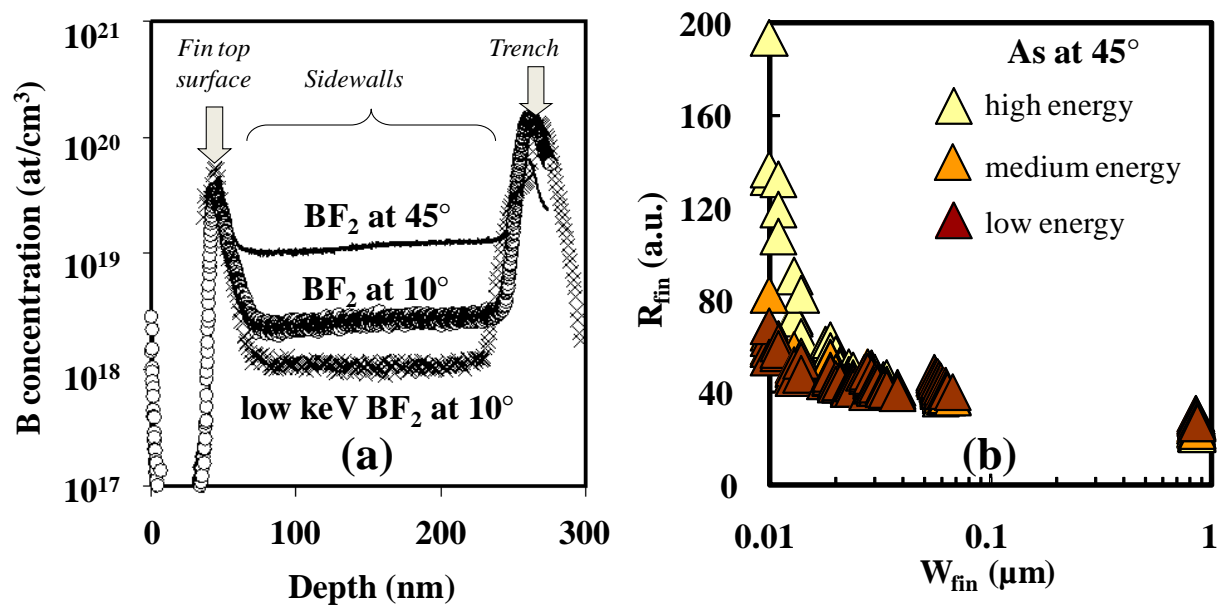

Fig. 22. (a) Apparent B concentrations obtained with SIMS analysis through a fin array. The plateau indicates sidewall doping. Low-energy low-tilt implants produce poor dose retention. (b) Experimental fin resistance $\left(R_{\text {fin }}\right)$ versus fin width $\left(W_{\text {fin }}\right)$ determined from a resistor experiment. High implant energies at $45^{\circ}$ tilt implants significantly increase fin resistance which evidences poor dopant incorporation (from Ref. [153]). 
In conclusion, the optimization of dopant profiles in fin devices implies a careful choice of tilt angle (as close to $45^{\circ}$ as possible but avoiding shadowing), implant energy (to introduce dopants into the sidewalls but without losing ions through the opposite side of the fin) and implant dose (to compensate for inefficient dopant incorporation, but avoiding significant target erosion).

\section{Doping conformality}

Another challenge that concerns dopant incorporation in fin structures is the conformality of the dopant distribution in the fin, i.e. the need to have similar doping characteristics throughout the fin region (top and sidewalls). Conformality affects the resistance of the $S / D$ regions since the total resistance in a region with a conformal profile would be smaller than that corresponding to a region with a non-conformal profile [168]. In addition, the loss of conformality leads to differences in turn-on behavior and current flow on the top and sidewalls of the fin [164] and increased gate-induced drain leakage currents (a major source of off-state leakage in FinFETs) [161,169].

The use of ion implantation complicates conformality of the dopant distribution in the fin as expected from the tilt angle limitations previously mentioned to avoid shadowing in closely spaced fins. Due to trigonometrical considerations, the dose irradiated in the sidewalls is a factor of $\tan (\alpha)$ with respect to the dose in the fin top [164]. Moreover, when using an implantation process with a tilt angle different from zero to dope both sidewalls of the fin (using the dual quadrant implant procedure shown in Fig. 18(b)), the sides of the fin are implanted with a single dose value whereas the top of the fin is implanted with a double dose value. In addition, as shown in Fig. 20, backscattering effects may also affect dopant conformality since the top and sidewalls of the fin are implanted with complementary angles. As an example, in Fig. 23 the KMC simulated dopant concentrations obtained for B implants with (a) $0.5 \mathrm{keV} 10^{15} \mathrm{~cm}^{-2}$ at $10^{\circ}$, (b) 2 $\mathrm{keV} 10^{15} \mathrm{~cm}^{-2}$ at $10^{\circ}$, (c) $0.5 \mathrm{keV} 5 \times 10^{15} \mathrm{~cm}^{-2}$ at $10^{\circ}$, and (d) $0.5 \mathrm{keV} 10^{15} \mathrm{~cm}^{-2}$ at $45^{\circ}$, in $10 \mathrm{~nm}$ wide fins, are shown (projected in a $x y$ cross section of the fin as schematized in Fig. 18(a)). Note that in these simulations not only the sidewall but also the top implant is considered, from both sides of the fin (as in the scheme of Fig. 18(b)). For $10^{\circ}$ implants, both conformality of the dopant distribution throughout the fin and the concentration of dopants incorporated into the sidewalls of the fin are poor. The geometrical factor alone already decreases the irradiated dose on the sidewalls in a factor of $1 / 2 \tan \left(10^{\circ}\right) \approx 0.08$ of 
the irradiated dose at the top surface, which complicates conformality. In addition, the inefficient dopant incorporation in the sidewalls ( $80^{\circ}$ implant angle) due to backscattering also favors the non-conformal doping profile in the fin. The ion loss is more pronounced for $0.5 \mathrm{keV}$ (90\% of the incident ions are backscattered and only 10\% retained) than for $2 \mathrm{keV}$ (30\% of the incident ions are retained, 65\% are backscattered, and there is an additional effect of about $5 \%$ of the ions that are lost because their trajectories escape from the fin). Increasing the implant dose at $0.5 \mathrm{keV}$ from $10^{15}$ to $5 \times 10^{15} \mathrm{~cm}^{-2}$ results in higher dopant concentrations but the poor level of conformality is maintained. For $45^{\circ}$ implants, the conformality and the dopant incorporation are best since $\tan \left(45^{\circ}\right)=1$ and about $60 \%$ of incident ions are retained. The irradiated dose on the sidewalls is now a factor of 0.5 of the irradiated dose at the top surface.

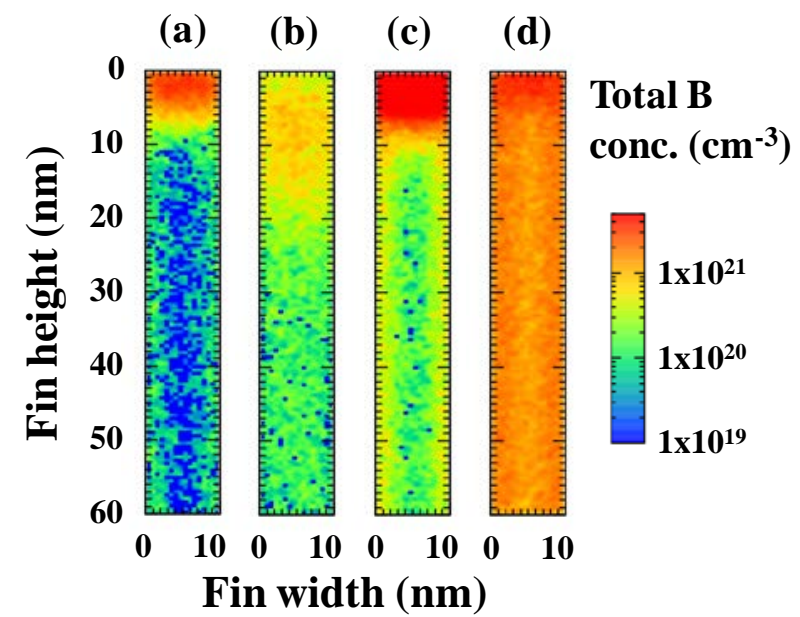

Fig. 23. 2D cross-section for dopant concentration in a $10 \mathrm{~nm}$ wide fin implanted with $B$ at (a) $0.5 \mathrm{keV}, 10^{15} \mathrm{~cm}^{-2}, 10^{\circ}$; (b) $2 \mathrm{keV}, 10^{15} \mathrm{~cm}^{-2}, 10^{\circ}$; (c) $0.5 \mathrm{keV}, 5 \times 10^{15} \mathrm{~cm}^{-2}, 10^{\circ}$; and (d) $0.5 \mathrm{keV}, 10^{15} \mathrm{~cm}^{-2}, 45^{\circ}$. A highly non-conformal dopant profile is obtained for $10^{\circ}$ implants (from Ref. [170]).

These simulation results are similar to those reported by Kambham et al. by APT measurements [23,167]. Figure 24 provides a comparison between the sidewall and top doping profiles obtained for a $200 \mathrm{~nm}$ tall fin array $\mathrm{BF}_{2}$ implanted at $5 \mathrm{keV}, 8 \times 10^{14} \mathrm{~cm}^{-2}$, with two different tilt angles $\left(10^{\circ}\right.$ and $\left.45^{\circ}\right)$. In the case of the $10^{\circ}$ implantation, dopants are shallower and significantly reduced in concentration for sidewall compared to top implant, showing a high non-conformality. For the $45^{\circ}$ tilt implantation dopants are deeper, and the top dopant concentration is around twice when compared to the sidewall dopant concentration. 


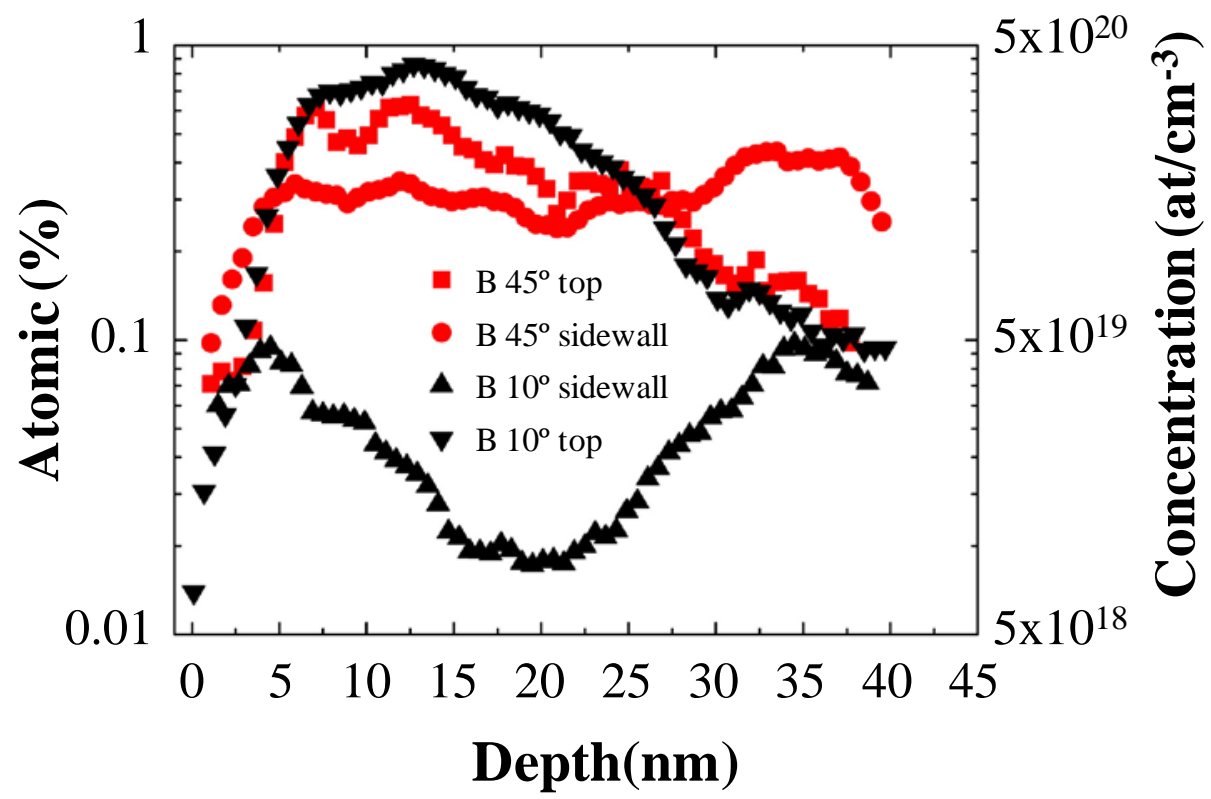

Fig. 24. Comparison between top and sidewall profiles for $10^{\circ}$ and $45^{\circ}$ implants as extracted from APT measurements (from Ref. [167]).

Similar trends have been observed by 2D scanning spreading resistance microscopy (SSRM) measurements (for the same experimental conditions) which provide 2D quantitative carrier profiles. This parameter is more relevant in terms of the evaluation of parasitic resistance than the dopant concentration, since it is the electrical behavior of dopants which determines $R_{S D}$. In order to improve electrical activation of dopants, a $900^{\circ} \mathrm{C}$ annealing was performed to dissolve formed boron-interstitial clusters (BICs), known to be responsible for B deactivation [171,172]. Again a clearly worst level of conformality of electrically active dopants is observed in the $10^{\circ}$ implantation case compared to the $45^{\circ}$ implantation case, as it is shown in Fig. 25. This behavior is also reproduced by atomistic KMC simulations. As an example, in Fig. 26 we show cross sections of both (a,c) crystalline or (b,d) preamorphized Si fins implanted with B ions with (a,b) $10^{\circ}$ and (c,d) $45^{\circ}$ tilted implants $\left(1 \mathrm{keV}, 8 \times 10^{14} \mathrm{~cm}^{-2}\right)$ and followed by a spike anneal at $1050^{\circ} \mathrm{C}$ to electrically activate dopants. As it occurred in conventional planar devices, higher levels of dopant activation can be achieved using preamorphizing implants [173]. As revealed by experiments, simulations evidence a significantly higher conformal distribution for electrically active $\mathrm{B}$ atoms in the $45^{\circ}$ implantation case compared to the $10^{\circ}$ implantation case. It is worthy to note that a proper modeling of the phenomenon of boron-interstitial clustering is required in order to accurately predict the 
concentration of electrically active B atoms. As discussed in a previous work, this becomes a difficult task as high B concentrations are concerned in aggressively scaled devices [171,172]. Experiments that involve high B concentrations in both crystalline Si $[174,175]$ and preamorphized Si [176,177] revealed the formation of BICs larger and more stable than expected from classical BICs models. In order to be able to reproduce these observations, the model for BICs was improved and its validity was demonstrated for low and high B concentration regimes in crystalline or preamorphized Si [171,172]. Results show that, at low and medium B concentrations $\left(>10^{20} \mathrm{~cm}^{-3}\right)$, classical models for BICs are capable of reproducing experimental data on B clustering and dissolution (thus correctly predicting B electrical activation). However, at higher B concentrations a fraction of small BICs evolve into larger configurations (experimentally visible by high resolution transmission electron microscopy) which can be very stable in preamorphized Si when low Si interstitial supersaturation levels exist. The formation of these highly stable BICs complicates their dissolution, thus slowing down B electrical activation.

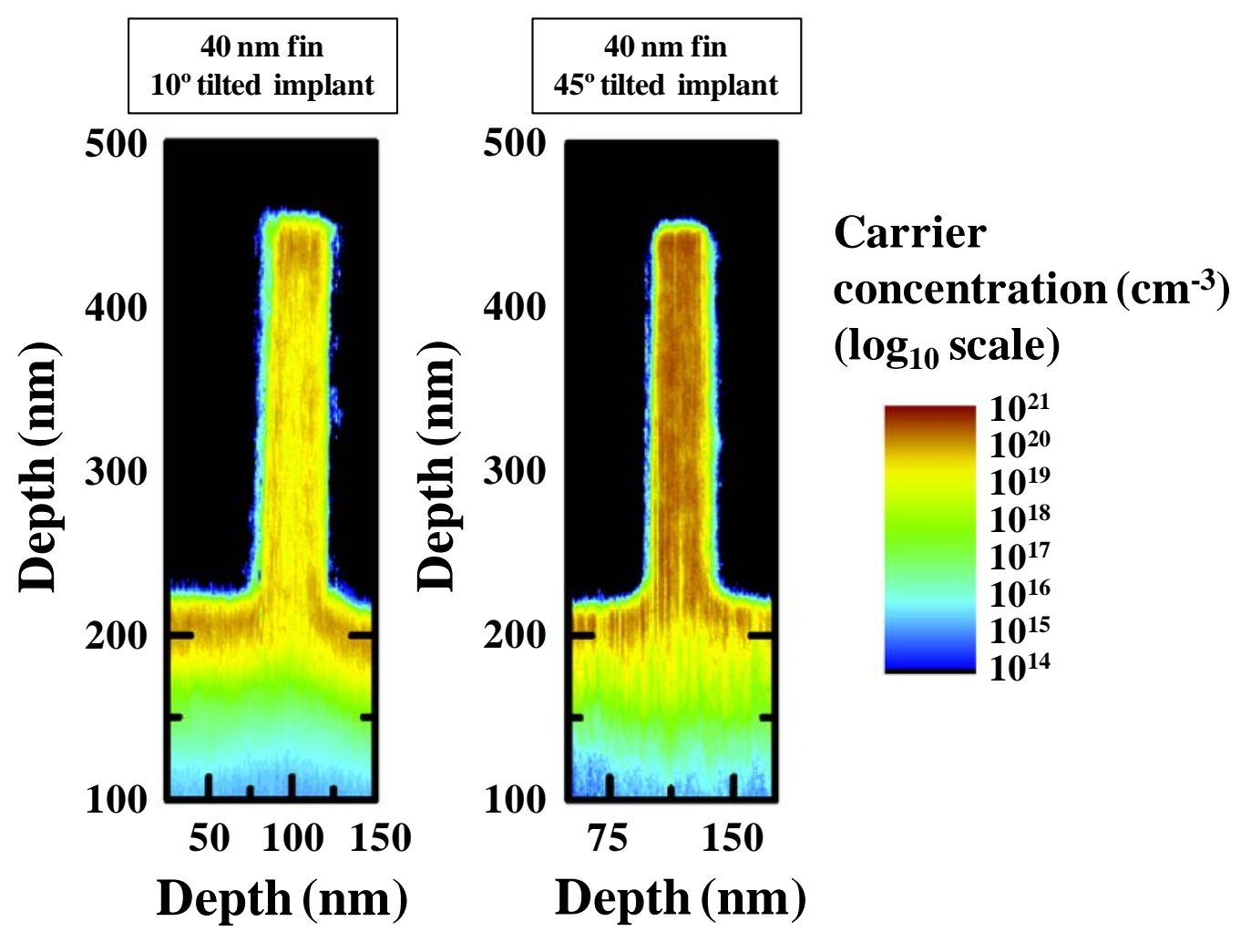

Fig. 25. 2D SSRM map of active carrier concentration of $\mathrm{BF}_{2}$ implanted fin at $10^{\circ}$ and $45^{\circ}$ implant angles and annealed at $900^{\circ} \mathrm{C}$ (from Ref. [178]). A higher level of conformality is obtained for a $45^{\circ}$ tilted implant. 
Crystalline substrate
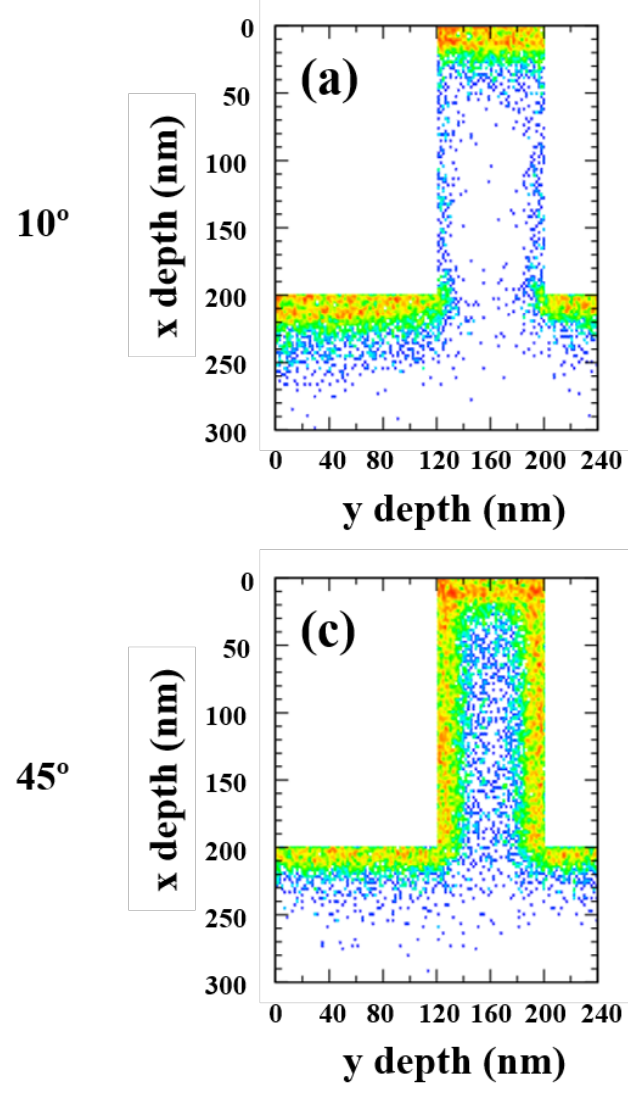

Pre-amorphized substrate

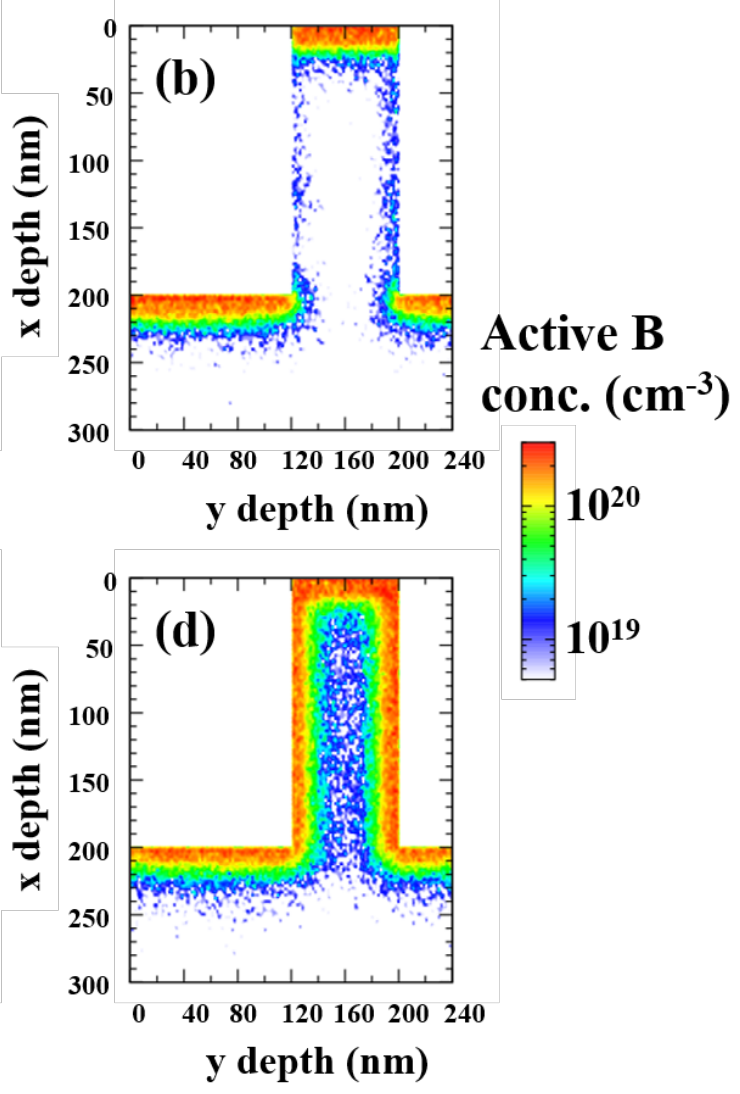

Fig. 26. Simulated electrically active $B$ concentration in $(a, c)$ crystalline or (b,d) preamorphized Si fins implanted with B ions with (a,b) $10^{\circ}$ and (c,d) $45^{\circ}$ tilt angles (1 $\mathrm{keV}, 8 \times 10^{14} \mathrm{~cm}^{-2}$ ) and spike annealed at $1050^{\circ} \mathrm{C}$. Higher level of conformality for active dopants is obtained for $45^{\circ}$ tilted implants in both crystalline and preamorphized $\mathrm{Si}$.

Thermal budget required for dopant activation as well as for defect removal also enhances dopant diffusion. In classical planar devices the minimization of sheet resistance along with the good SCE control requirements implied a trade-off between high dopant activation (degraded by the formation of dopant-defect clusters such as BICs) and minimal junction depth (degraded by dopant diffusion) [179]. However, in a FinFET device the situation is quite different. The S/D extension junction depth is controlled by the small dimensions of the fin. Concerning the S/D implants, in SOI FinFETs the vertical junction depth is no longer a relevant parameter (as it is rather set by the Si thickness) and only the lateral profile characteristics are relevant [23]. In fact, the lateral diffusion of the dopants into the channel under the gate (gate overlap) is an important parameter that affects the final device performance [160]. This lateral diffusion has been also analyzed by KMC simulations in Fig. 27. For this purpose, several B implants with the same 
conditions as in Fig. 23 were performed and followed by a thermal annealing process. In this case B concentrations in a $x z$ cross section (dashed slice in the scheme shown in Fig. 18(a)) were evaluated, providing a lateral view of dopants as-implanted and after a $1100^{\circ} \mathrm{C}$ spike anneal step. Simulations show that dopants diffuse during annealing, penetrating from the S/D region into the channel for all implant conditions. It also clearly evidences that under-diffusion varies along the height of the fin, especially for the $10^{\circ}$ tilted implants. Thus, a higher lateral diffusion is obtained in the top of the fin compared to the bottom of the fin (due to the higher concentration of dopants in the top compared to sidewalls). This effect is more pronounced for low angle tilt implants due to the high non-conformality of dopants observed in Fig. 23. Similar behavior for lateral diffusion was later confirmed by 3D APT measurements performed by Vandervorst et al. [23] in As plasma-doped fins, as it is illustrated in Fig. 28. The authors observed lateral diffusion of dopants into the channel under the gate which is enhanced at the top of the fin. They attributed this enhancement to the non-conformal doping used in their measurements causing higher dopant incorporation in the top of the fin and thus leading to more lateral diffusion.

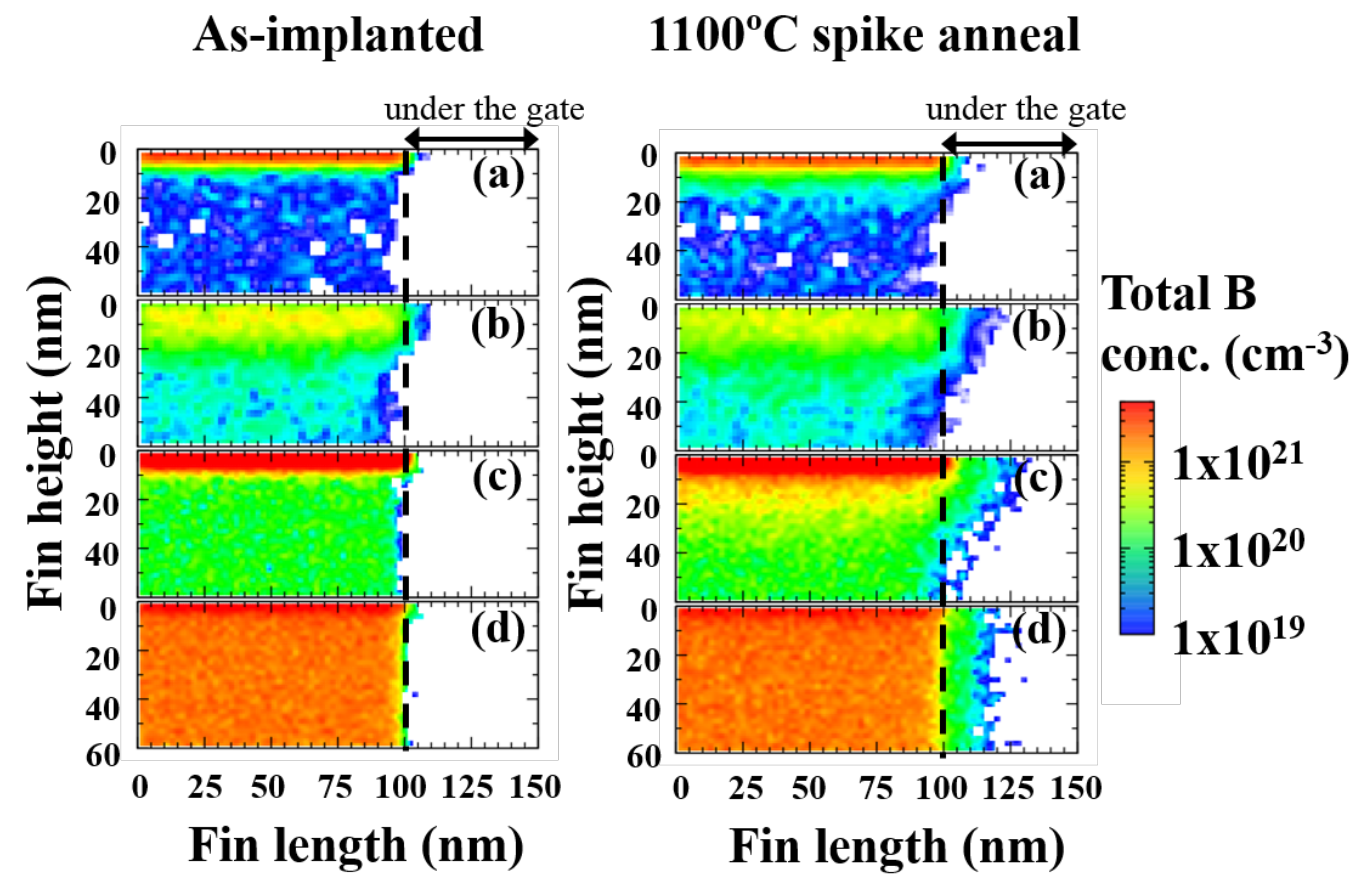

Fig. 27. Lateral view of the $B$ concentration in a FinFET implanted with $B$ at (a) $0.5 \mathrm{keV}$, $10^{15} \mathrm{~cm}^{-2}, 10^{\circ}$; (b) $2 \mathrm{keV}, 10^{15} \mathrm{~cm}^{-2}, 10^{\circ}$; (c) $0.5 \mathrm{keV}, 5 \times 10^{15} \mathrm{~cm}^{-2}, 10^{\circ}$; and (d) $0.5 \mathrm{keV}$, $10^{15} \mathrm{~cm}^{-2}, 45^{\circ}$; as implanted (left) and after a $1100^{\circ} \mathrm{C}$ spike anneal (right) (from Ref. $[170])$. 


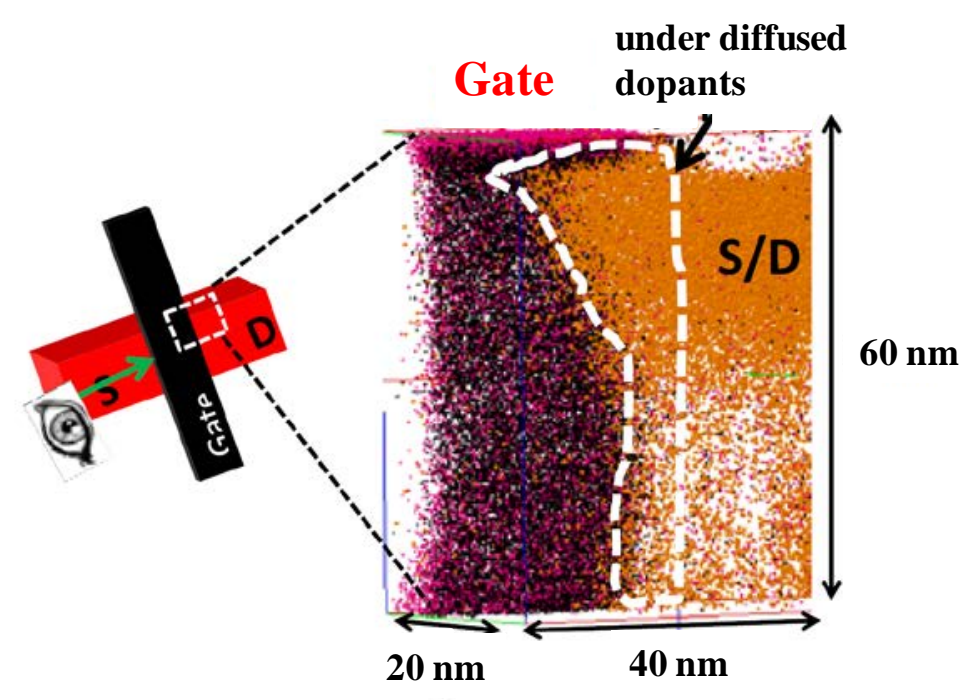

Fig. 28. 3D doping profile obtained with APT for As plasma doping and diffusion from the S/D region to the gate (from Ref. [23]). Slice taken along the direction indicated in the insert.

As an alternative to beam directed ion implantation, isotropic doping techniques like plasma-immersion doping (PLAD) [180] and improved self-regulatory plasma doping [159] can be used to obtain conformal profiles throughout the fin. High levels of conformality have been obtained for p-type doping of Si fins [159,181]. Nevertheless, conformal doping is more difficult for n-type doping. This is due to the low adsorption efficiency of n-type dopants on Si surfaces that results in poor incorporation of As or $\mathrm{P}$ on the fin sidewalls $[18,19,160,162,180]$. Dopants located at the fin surface are then vulnerable to subsequent resist strip and etching processes (i.e. dopant losses for As doped fins from $75 \%$ [180] to a recently improved value of around $25 \%$ after the strip process have been reported [162]). To avoid dopant loss after the strip process, recent studies considered the use of a rapid thermal annealing (RTA) after plasma doping in order to drive the dopants inside the fin (in-diffusion) and electrically activate them [18,19]. As an example, Fig. 29(a) displays a SSRM image of a plasma As doped fin RTA annealed at $1050^{\circ} \mathrm{C}$, showing good doping conformality along the top and sidewalls of the fin [161]. Additionally, plasma doped Si lattice becomes less damaged compared to ion implanted Si lattice [161]. As discussed in Section 3, ion implantation induces poor recrystallization in narrow fins leading to defect formation, which is avoided by plasma doping (see Fig. 29(b) and (c)). Nevertheless, although the use of RTA reduces dopant loss, dopant deactivation and pile-up/segregation at trap sites at the interface layers (thus 
affecting $R_{S D}$ ) as well as significant gate overlap (thus degrading SCE) were observed due to the increased thermal budget [18]. More recently, Lee et al. have considered the deposition of an oxide buffer layer prior to plasma doping instead of RTA, achieving about $76 \%$ of the As doped concentration retained in the Si fin after the strip process and even after ashing [162]. Nevertheless, although recent works on plasma doping in FinFETs are promising in terms of dopant incorporation and conformality, this technique is still quite challenging. Its implementation is compromised by concurrent artifacts, sputter erosion being the most important one, especially for narrow fins. In fact, a tradeoff exists between conformal doping and fin erosion [19]. This may lead to significant fin deformation (especially at corners) and it also complicates dopant incorporation, thus degrading device characteristics. Recently, trap generation on the surface of the fin has also been reported, which aggravates both on- and off-state characteristics [162].
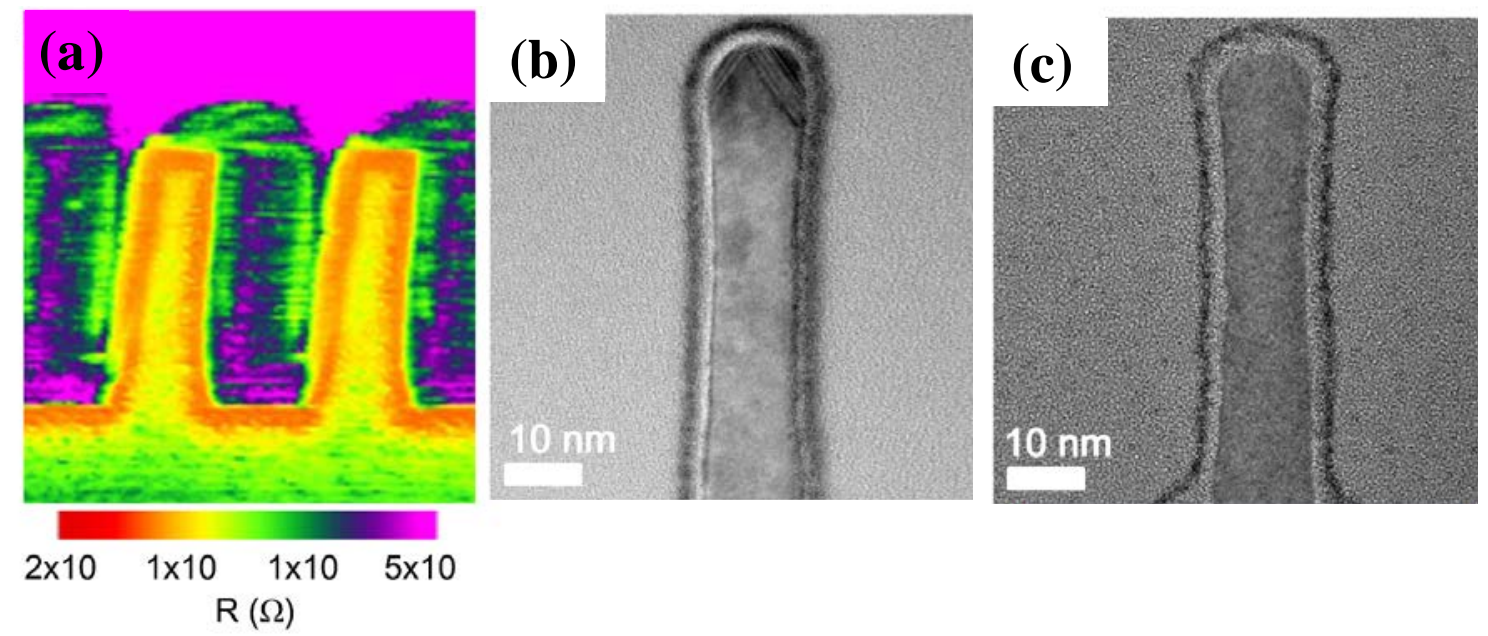

Fig. 29. (a) SSRM image of conformal plasma doped fin; Cross-sectional transmission electron microscopy images of fins doped by (b) ion implantation and (c) plasma doping (from Ref. [161]).

In addition, modeling plasma doping is extremely difficult since this process is inherently a balance of several mechanisms including implantation, deposition, knock-in and surface sputtering/etching [180,182-185]. The competition and balance among all these concurrent processes can be optimized by varying the large amount of plasma doping parameters such as energy, dose, power, bias pulsing, pressure and gas dilution ratios, in order to obtain the desired dopant profile characteristics [180]. Several factors determine the doping profiles resulting from plasma doping, including plasma chemistry, deposition and etching characteristics, and energy distribution in the collisional plasma 
[186]. Thus, a challenging objective could be the mapping of all the concurring phenomena in a reliable computational tool for the plasma doping simulation. Recently, some advances on plasma doping modeling have been obtained and applied to the simulation of dopant profiles in 3D devices [184,185]. Burenkov et al. analyzed dopant profiles in FinFETs by using the Monte Carlo PLAD model implemented in the Synopsis SENTAURUS process simulator [24,185], which is particularly focused on the prediction of ion penetration. In that simulation code, the PLAD process is modeled as a simple combination of conformal and directed implantation. Instead of launching all ions from above the device, as in beam-directed ion implantation, a fraction of ions is launched along the device surface by considering particular energy and angle distributions. However, as stated by La Magna et al., this approach takes into account only a limited part of the phenomena occurring during the plasma doping process, neglecting the role of neutrals adsorption, ion beam surface modifications (due to deposition and/or erosion) and the proper angular distribution of the plasma components [184]. These authors proposed a more sophisticated model which is able to predict fin erosion during plasma doping. Nevertheless, although the mapping of the different events is more complete in their implementation, La Magna and coworkers mentioned that further advancements are still needed for the parameter calibration and for the reliable coupling with the generic plasma conditions.

\section{Conclusions}

We have overviewed the pathway of physics-based modeling improvement through a multi-scale scheme where several techniques are combined to gain fundamental understanding that can guide the technology. Models have been improved to capture physical mechanisms in more detail as a response to the accuracy requested in modern process and device modeling. The demanding requirements for next generation Si integrated circuits have not only imposed the change from the traditional 2D planar FETs to 3D multigate MOS devices, but also a critical control of process parameters to limit device variability. In this scenario of complex geometry devices in the nanoscale where accurate process description is needed, atomistic methods become the most appropriate strategy to simulate even full-size structures. We have focused on the evolution of ion implantation and damage models to comply with the request of process technology. We have illustrated the role of implant parameters to minimize residual damage and we have 
addressed doping issues that arise in FinFETs, where atomistic simulation becomes an essential tool to complement the complex characterization techniques needed for device optimization.

Although detailed implant, defect and dopant models have been formulated to satisfy the demands of the fast evolving CMOS Si technology, other fields of the semiconductor industry such as solar cells, image sensors, and power devices also benefit from these developments. Even other areas of application such as devices exposed to radiation (nuclear detectors, or devices in space) take advantage of the improved understanding of irradiation cascades and damage cure.

\section{Acknowledgements}

This work has been supported by EU (FEDER) and the spanish Ministerio de Ciencia e Innovación under Project No. TEC2014-60694-P, and by the Junta de Castilla y León under Project No. VA331U14.

\section{References}

[1] K. J. Kuhn, IEEE Trans. Electron Devices 59 (2012) 1813.

[2] Y. Cui, Z. Zhong, D. Wang, W. U. Wang, C. M. Lieber, Nano Lett. 3 (1982) 149.

[3] J. L. Liu, Y. Lu, Y. Shi, S.L. Gu, R. L. Jiang, F. Wang, Y. D. Zheng, Appl. Phys. A 66 (1998) 539.

[4] C. Ronning, C. Borschel, S. Geburt, R. Niepelt, Mater. Sci. Eng. R. 70 (2010) 30.

[5] See http://www.itrs.net/ for International Technology Roadmap for Semiconductors.

[6] C. Auth et al., in: VLSI technology (VLSIT), 2012 symposium on, 12-14 June 2012. pp. 131-132.

[7] S. Natarajan et al., 2014 IEEE International Electron Devices Meeting, San Francisco, CA, 2014, pp. 3.7.1-3.7.3.

[8] X. Wei, H. Zhu, Y. Zhang, C. Zhao, Solid-State Electron. 122 (2016) 45.

[9] C.-W. Lee, S.-R.-N. Yun, C.-G. Yu, J.-T. Park, J.-P. Colinge, Solid-State Electron. 51 (2007) 505.

[10] I. Ferain, C. A. Colinge, J. -P. Colinge, Nature 479 (7373) (2011) 310. 
[11] Y. Guerfi, G. Larrieu, Nanoscale Res. Lett. 11 (2016) 210.

[12] J. Appenzeller, J. Knoch, M. T. Björk, H. Riel, H. Schmid, W. Riess, IEEE Trans. Electron Devices 55 (2008) 2827.

[13] J. Fan, M. Li, X. Xu, Y. Yang, H. Xuan, R. Huang, IEEE Trans. Electron Devices 62 (2015) 213.

[14] B. -H. Lee, M. -H. Kang, D. -C. Ahn, J. -Y. Park, T. Bang, S. -B. Jeon, J. Hur, D. Lee, Y. -K. Choi, Nano Lett. 15 (2015) 8056.

[15] B. -H. Lee, J. Hur, M. -H. Kang, T. Bang, D. -C. Ahn, D. Lee, K. -H. Kim, Y. -K. Choi, Nano Lett. 16 (2016) 1840.

[16] E. Chason, S. T. Picraux, J. M. Poate, J. O. Borland, M.I. Current, T. D. de la Rubia, D. J. Eaglesham, O. W. Holland, M. E. Law, C. W. Magee, J. W. Mayer, J. Melngailis, A. F. Tasch, J. Appl. Phys. 81 (1997) 6513.

[17] A. Rohatgi, D. L. Meier, B. McPherson, Y.-W Ok, A. D. Upadhyaya, J. H. Lai F. Zimbardi, Energy Procedia 15 (2012) 10.

[18] A. K. Kambham, A. Kumar, A. Florakis, W. Vandervorst, Nanotechnology 24 (2013) 275705.

[19] Y. Sasaki, K. Okashita, B. Mizuo, M. Kubota, M. Ogura, O. Nishijima, J. Appl. Phys. 111 (2012) 013712.

[20] D. J. Eaglesham, P. A. Stolk, H. -J. Gossman, J. M. Poate, Appl. Phys. Lett. 65 (1994) 2305.

[21] J.-Y. Jin, J. Liu, U. Jeong, S. Mehta, K.S. Jones, J. Vac. Sci. Technol. B 20 (2002) 422.

[22] M. Leong, B. Doris, J. Kedzierski, K. Rim, M. Yang, Science 306 (2004) 2057.

[23] W. Vandervorst, A. Schulze, A. K. Kambham, J. Mody, M. Gilbert, Pierre Eyben, Phys. Status Solidi C 11 (2014) 121.

[24] Sentaurus ${ }^{\mathrm{TM}}$ Process User Guide, I-2013.12, Synopsys, Inc., Mountain View, CA 94043, USA, December 2013.

[25] Zwanenburg F.A., Dzurak A.S., Morello A. Simmons M.Y. Hollenberg L.C. L. Klimerk G. et al., Rev. Mod. Phys. 85 (2013) 961.

[26] FLOOPS: FLorida Object Oriented Device and Process Simulator. See http://www.flooxs.tec.ufl.edu/.

[27] M. Jaraiz, L. Pelaz, J. E. Rubio, J. Barbolla, G. H. Gilmer, D. J. Eaglesham, H. J. Gossman, J. M. Poate, Mater. Res. Soc. Symp. Proc. 532 (1998) 43. 
[28] See http://www.materials.imdea.org/MMonCa for the Modular Monte Carlo simulator.

[29] L. A. Marqués, L. Pelaz, J. Hernández, J. Barbolla, G.H. Gilmer, Phys. Rev. B 64 (2001) 45214.

[30] L. A. Marqués, I. Santos, L. Pelaz, P. López, M. Aboy, Mater. Sci. Semicond. Process. 42 (2016) 235.

[31] N. A. Modine, A.F. Wright, S.R. Lee, Comput. Mater. Sci. 92 (2014) 431.

[32] C. Freysoldt, B. Grabowski, T. Hickel, J. Neugebauer, G. Kresse, A. Janotti, C. G. Van de Walle, Rev. Mod. Phys. 86 (2014) 253.

[33] Y. Qiu, F. Cristiano, K. Huet, F. Mazzamuto, G. Fisicaro, A. La Magna, M. Quillec, N. Cherkashin , H. Wang, S. Duguay, D. Blavette, Nano Lett. 14 (4) (2014) 1769.

[34] R.G. Elliman, J.S. Williams, Current opinion in Solid State and Materials Science 19 (2015) 49.

[35] N. Zographos, C. Zechner, I. Martín-Bragado, K. Lee, Y-S. Oh, "Multiscale modeling of doping processes in advanced semiconductor devices”, (this issue).

[36] L. Pelaz, L. A. Marqués, M. Aboy, P. López, I. Santos, Eur. Phys. J. B 72 (2009) 323.

[37] M. T. Robinson, I. M. Torrens, Phys. Rev. B 9 (1974) 5008.

[38] J. F. Ziegler, Ion Implantation: Science and Technology (Academic Press, San Diego, 1988)

[39] H. Goldstein, Classical Mechanics, 3rd edn. (Addison-Wesley, Reading, MA, 2000)

[40] UT-MARLOWE documentation, http://homer.mer.utexas.edu/

[41] Y. Chen, G. Wang, D. Li, S. K. Oak, G. Shrivastav, L. Rubin, A. F. Tasch, S. K. Banerjee, IEEE Trans. Electron Devices 49 (2002) 1519.

[42] J. M. Hernández-Mangas, J. Arias, L. Bailón, M. Jaraiz, J. Barbolla, J. Appl. Phys. 91 (2002) 658.

[43] SRIM documentation, http://www.srim.org

[44] J. J. Loferski, P. Rappaport, Phys. Rev. 98 (1955) 1861.

[45] L. A. Miller, D. K. Brice, A. K. Prinja, S. T. Picraux, Phys. Rev. B 49 (1994) 16953.

[46] E. Hölmstrom, A. Kuronen, K. Nordlund, Phys. Rev. B 78 (2008) 045202.

[47] P. Sigmund, Appl. Phys. Lett. 14 (1969) 114. 
[48] See for example the description of the "Kinchin-Pease Damage Model" of UTMARLOWE, http://homer.mer.utexas.edu

[49] E. Landi, A. Armigliato, S. Solmi, R. Kögler, E. Wieser, Appl. Phys. A 47 (1988) 359.

[50] M. H. Clark, K. S. Jones, T. E. Haynes, C. J. Barbour, K. G. Minor, E. Andideh, Appl. Phys. Lett. 80 (2002) 4163.

[51] Y. Kawasaki, T. Kuroi, T. Yamashita, K. Horita, T. Hayashi, M. Ishibashi, M. Togawa, Y. Ohno, M. Yoneda, T. Horsky et al., Nucl. Instrum. Methods Phys. Res. B 237 (2005) 25.

[52] J. M. Haile, “Molecular Dynamics Simulations: Elementary Methods”, John Wiley \& Sons, 1992, New York.

[53] J. F. Ziegler, J. P. Biersack, U. Littmark, "The Stopping and Range of Ions in Solids” (Pergamon, New York, 1985).

[54] M. J. Caturla, T. D. de la Rubia, L. A. Marqués, G. H. Gilmer, Phys. Rev. B 54 (1996) 16683.

[55] G. Hobler, G. Otto, Mater. Sci. Semicond. Process. 6 (2003) 1.

[56] I. Santos, L. A. Marqués, L. Pelaz, P. López, M. Aboy, J. Barbolla, Mater. Sci. Eng. B 124-125 (2005) 372.

[57] T. Aoki, J. Matsuo, G. Takaoka, N. Toyoda, I. Yamada, Nucl. Instrum. Methods Phys. Res. B 206 (2003) 855.

[58] L. A. Marqués, L. Pelaz, I. Santos, V. C. Venezia, Phys. Rev. B 74 (2006) 201201.

[59] T. Aoki, J. Comput. Electron. 13 (2014) 108.

[60] K. Nordlund, F. Djurabekova, J. Comput. Electron. 13 (2014) 122.

[61] G. Hobler, G. Otto, Nucl. Instrum. Methods Phys. Res. B 206 (2003) 81.

[62] D. Kovac, G. Otto, G. Hobler, Nucl. Instrum. Methods Phys. Res. B 228 (2005) 226.

[63] I. Santos, L. A. Marqués, L. Pelaz, Phys. Rev. B 74 (2006) 174115.

[64] D. Kovac, G. Hobler, Nucl. Instrum. Methods Phys. Res. B 267 (2009) 1229.

[65] I. Santos, L. A. Marqués, L. Pelaz, P. López, M. Aboy, Solid State Phenomena 139 (2008) 71.

[66] I. Santos, L. A. Marqués, L. Pelaz, P. López, J. Appl. Phys. 105 (2009) 083530.

[67] M. J. Beck, R. D. Schrimpf, D. M. Fleetwood, S. T. Pantelides, Phys. Rev. Lett. 100 (2008) 185502. 
[68] P. López, L. Pelaz, I. Santos, L. A. Marqués, M. Aboy, J. Appl. Phys. 111 (2012) 033519.

[69] C. Claeys, E. Simoen, “Germanium-Based Technologies - From Materials to Devices”, (Elsevier, Amsterdam, 2007)

[70] M. Caymax, G. Eneman, F. Bellenger, C. Merckling, A. Delabie, G. Wang, R. Loo, E. Simoen, J. Mitard, B. de Jaeger, G. Hellings, K. de Meyer, M. Meuris, M. Heyns, IEDM Tech. Dig. (2009) 428-431.

[71] P. Kurczynski, R. H. Pehl, E. L. Hull, D. Palmer, M. J. Harris, H. Seifert, B. J. Teegarden, N. Gehrels, T. L. Cline, R. Ramaty, D. Sheppard, N. W. Madden, P. N. Luke, C. P. Cork, D. A. Landis, D. F. Malone, K. Hurley, Nucl. Instrum. Methods Phys. Res. A 431 (1997) 141.

[72] A. Owens, S. Brandenburg, E.-J. Buis, H. Kiewiet, S. Kraft, R. W. Ostendorf, A. Peacock, F. Quarati, P. Quirin, Nucl. Instrum. Methods Phys. Res. A 583 (2007) 285.

[73] S. Koffel, P. Scheiblin, A. Claverie, G. BenAssayag, J. Appl. Phys. 105 (2009) 013528.

[74] R. J. Kaiser, S. Koffel, P. Pichler, A. J. Bauer, B. Amon, A. Claverie, G. BenAssayag, P. Scheiblin, L. Frey, H. Ryssel, Thin Solid Films 518 (2010) 2325.

[75] J. A. Van den Berg et al., J. Vac. Sci. Technol. B 20 (2002) 974.

[76] J. A. van den Berg, S. Zhang, S. Whelan, D. G. Armour, R. D. Goldberg, P. Bailey, T. C. Q. Noakes, Nucl. Instrum. Methods Phys. Res. B 183 (2002) 154.

[77] L. Rubin, J. Poate, The Industrial Physicist 9 (2003) 12.

[78] J. A. van den Berg, G. Carter, D. G. Armour, M. Werner, R. D. Goldberg, E. J. H. Collart, P. Bailey, T. C. Q. Noakes, Appl. Phys. Lett. 85 (2004) 3074.

[79] H. Hensel, H. M. Urbassek, Phys. Rev. B 57 (1998) 4756.

[80] J. Tarus, K. Nordlund, A. Kuronen, J. Keinonen, Phys. Rev. B 58 (1998) 9907.

[81] E. Hölmstrom, L. Toikka, A. V. Krasheninnikov, K. Nordlund, Phys. Rev. B 82 (2010) 045420.

[82] S. Hoilijoki, E. Hölmstrom, K. Nordlund, J. Appl. Phys. 110 (2011) 043540.

[83] M. Y. Tsai, B. G. Streetman, J. Appl. Phys. 50 (1979) 183.

[84] L. Csepregi, E. F. Kennedy, J. W. Mayer, T. W. Sigmon, J. Appl. Phys. 49 (1978) 3906.

[85] K. S. Jones, K. Moller, J. Chen, M. Puga-Lambers, B. Freer, J. Berstein, L. Rubin, J. Appl. Phys. 81 (1997) 6051. 
[86] D. R. Lim, C. S. Rafferty, F. P. Klemens, Appl. Phys. Lett. 67 (1995) 2302.

[87] R. Duffy, M. J. H. Van Dal, B. J. Pawlak, M. Kaiser, R. G. B. Degroote, E. Kunnen, E. Altamirano, Appl. Phys. Lett. 90 (2007) 241912.

[88] M. J. H. van Dal, N. Collaert, G. Doornbos, G. Vellianitis, G. Curatola, B. Pawlak, R. Duffy, C. Jonville, B. Degroote, E. Altamirano, E. Kunnen, M. Demand, S. Beckx, T. Vandeweyer, C. Delvaux, F. Leys, A. Hikavyy, R. Rooyackers, M. Kaiser, R. G. R. Weemaes, S. Biesemans, M. Jurczak, K. Anil, L. Witters, R. J. P. Lander, in Dig. Tech. Pap. - Symp. VLSI Technol. (2007) 110-111.

[89] F. L. Vook, in Radiation Damage and Defects in Semiconductors, Inst. Phys. Conf. Ser. No. 16 p. 60, edited by J. E. Whitehouse (Institute of Physics, London, 1973).

[90] J. R. Dennis, E. B. Hale, J. Appl. Phys. 49 (1978) 1119.

[91] L. Pelaz, L. A. Marqués, M. Aboy, J. Barbolla, Defect and Diffusion Forum 221 (2003) 31.

[92] L. Pelaz, L. A. Marqués, J. Barbolla, J. Appl. Phys. 96 (2004) 5947.

[93] J. E. Rubio, M. Jaraiz, I. Martín-Bragado, R. Pinacho, P. Castrillo, J. Barbolla, Mater. Sci. Eng. B 114 (2004) 151.

[94] I. Martin-Bragado, V.Moroz, Appl. Phys. Lett. 95 (2009) 123123.

[95] S. Prussin, D. I. Margolese, R. N. Tauber, J. Appl. Phys. 57 (1985) 180.

[96] M. L. Swanson, J. R. Parsons, C. W. Hoelke, Radiat. Eff. 9 (1971) 249.

[97] I. Avci, M.E. Law, E. Kuryliw, K.S. Jones, IEDM Tech. Dig. (2001) 835.

[98] M. Tang, L. Colombo, J. Zhu, T. D. de la Rubia, Phys. Rev. B 55 (1997) 14279.

[99] D. M. Stock, B. Weber, K. Gärtner, Phys. Rev. B 61 (2000) 8150.

[100] F. Cargnoni, C. Gatti, L. Colombo, Phys. Rev. B 57 (1998) 170.

[101] S. Goedecker, T. Deutsch, L. Billard, Phys. Rev. Lett. 88 (2002) 235501.

[102] L. A. Marqués, L. Pelaz, I. Santos, P. López, R. Duffy, J. Appl. Phys. 111 (2012) 034302.

[103] L. A. Marqués, L. Pelaz, P. López, I. Santos, M. Aboy, Phys. Rev. B 76 (2007) 153201.

[104] P. López, L. Pelaz, L. A. Marqués, I. Santos, J. Appl. Phys. 101 (2007) 093518.

[105] K. R. C. Mok, F. Benistant, M. Jaraiz, J.E. Rubio, P. Castrillo, R. Pinacho, M. P. Srinivasan, J. Appl. Phys. 103 (2008) 014911.

[106] R. Drosd, J. Washburn, J. Appl. Phys. 53 (1982) 397.

[107] N. Rudawski, K. Jones, Scr. Mater. 61 (2009) 327. 
[108] J. Custer, M. Thompson, D. Jacobson, J. Poate, S. Roorda, W. C. Sinke, F. Spaepen, Appl. Phys. Lett. 64 (1994) 437.

[109] E. Lampin, C. Krzeminski, J. Appl. Phys. 106 (2009) 063519.

[110] C. Krzeminski, E. Lampin, Eur. Phys. J. B 81 (2011) 283.

[111] M. Prieto-Depedro, I. Romero, I. Martin-Bragado, Acta Mater. 82 (2015) 115.

[112] I. Martin-Bragado, V. Moroz, Appl. Phys. Lett. 98 (2011) 153111.

[113] I. Martin-Bragado, A. Sarikov, Mater. Sci. Semicond. Process. 42 (2016) 223.

[114] I. Martín-Bragado, B. Sklenard, J. Appl. Phys. 112 (2012) 024327.

[115] L. Pelaz, M. Aboy, P. López, L. A. Marqués, J. Vac. Sci. Technol. B 24 (2006) 2432.

[116] P. F. Fazzini, F. Cristiano, C. Dupré, S. Paul, T. Ernst, H. Kheyrandish, K. K. Bourdelle, W. Lerch, Mater. Sci. Eng. B 256 (2008) 154.

[117] K. L. Saenger, J. P. de Souza, K. E. Fogel, J. A. Ott, C. Y. Sung, D. K. Sadana, H. Yin, J. Appl. Phys. 101 (2007) 024908.

[118] K. L. Saenger, K. E. Fogel, J. A. Ott, D. K. Sadana, H. Yin, J. Appl. Phys. 101 (2007) 104908.

[119] I. Ferain, R. Duffy, N. Collaert, M. J. H. van Dal, B. J. Pawlak, B. O’Sullivan, L. Witters, R. Rooyackers, T. Conard, M. Popovici, S. van Elshocht, M. Kaiser, R. G. R. Weemaes, J. Swerts, M. Jurczak, R. J. P. Lander, K. De Meyer, Solid-State Electron. 53 (2009) 760.

[120] R. A. Kelly, J. D. Holmes, N. Petkov, Nanoscale 6 (2014) 12890.

[121] T. Merelle, G. Curatola, G. Nackaerts, A. Collaert, M. J. H. van Dal, G. Doornbos, T. S. Doorn, P. Christie, G. Vellianitis, B. Duriez, R. Duffy, B. J. Pawlak, F. C. Voogt, R. Rooyackers, L. Witters, M. Jurczak, R. J. P. Lander, IEDM Tech. Dig. (2008) 241-244.

[122] E. Lampin, C. Krzeminski, J. Appl. Phys. 109 (2011) 123509.

[123] L. Csepregi, J. W. Mayer, T. W. Sigmon, Appl. Phys. Lett. 29 (1976) 92.

[124] K. F. Kelton, A. L. Greer, C. V. Thompson, J. Chem. Phys. 79 (1983) 6261.

[125] J. D. Plummer, M. D. Deal, P.B. Griffin, Silicon VLSI Technology. Fundamentals, Practice and Modeling (Prentice Hall, 2000).

[126] O. W. Holland, S. J. Pennycook, G. L. Albert, Appl. Phys. Lett. 55 (1989) 2503.

[127] G. Hobler, A. Simionescu, L. Pametshofer, C. Tian, G. Stingeder, J. Appl. Phys. 77 (1995) 3697.

[128] G. Hobler, Nucl. Instrum. Methods Phys. Res. B 96 (1995) 155. 
[129] J. Arias, M. Jaraiz, L. Pelaz, L. Bailón, J. Barbolla, Nucl. Instrum. Methods Phys. Res. B 102 (1995) 228.

[130] J. Arias, M. Jaraiz, J. E. Rubio, L. Pelaz, L. A. Marqués, J. Barbolla, J. Mater. Sci. Technol. 11 (1995) 1191.

[131] P. López, L. Pelaz, L. A. Marqués, J. Barbolla, H.-J.L. Gossmann, A. Agarwal, K. Kimura, T. Matsushita, Mater. Sci. Eng. B 124-125 (2005) 379.

[132] W. P. Maszara, G. A. Rozgonyi, J. Appl. Phys. 60 (1986) 2310.

[133] K. S. Jones, S. Prussin, E. R. Weber, Appl. Phys. A 45 (1988) 1.

[134] L. A. Larson, J. M. Williams, M. I. Current, Reviews of Accelerator Science and Technology, 4 (2011) 11.

[135] D. Jacobson, T. Horsky, W. Krull, B. Milgate, Nucl. Instrum. Methods Phys. Res. B 237 (2005) 406.

[136] R. G. Elliman, J. Linnros, W. L. Brown, in Fundamentals of Beam-Solid Interactions and Transient Thermal Processing, edited by Michael J. Aziz, Lynn E. Rehn, Bernd Stritzker, MRS Symposia Proceedings 100 (Materials Research Society, Pittsburgh, (1988) 363-368.

[137] M. S. Ameen, M. A. Harris, C. Huynh, R. N. Reece, Ion Implantation Technology AIP Conf. Proc. 1066 (2008) 30-33.

[138] J. Singer, M. Jaraiz, P. Castrillo, C. Laviron, N. Cagnat, F. Wacquant, O. Cueto, A. Poncet, Ion Implantation Technology AIP Conf. Proc. 1066 (2008) 209-212.

[139] H. J. Gossmann, N. Zographos, H. Park, B. Colombeau, T. Parrill, N. Khasgiwale, R. Borges, R. Gull, Y. Erokhin, Ion Implantation Technology AIP Conf. Proc. 1496 (2012) 225-228.

[140] K. Kimura, T. Matsushita, private communication.

[141] S. Prussin, D. I. Margolese, R. N. Tauber, J. Appl. Phys. 54 (1983) 2316.

[142] C. L. Yang, C. H. Tsai, C. I. Li, C. Y. Tzeng, G. P. Lin, W. J. Chen, Y. L. Chin, C. I. Liao, M. Chan, J. Y. Wu, E. R. Hsieh, B. N. Guo, S. Lu, B. Colombeau, S. S. Chung, I. C. Chen, IEEE Electron Dev. Lett. 33 (2012) 1444.

[143] C. L. Yang, C. I. Li, G. P. Lin, R. Liu, B. C. Hsu, M. Chan, J. Y. Wu, B. Colombeau, B. N. Guo, H.-J. Gossmann, T. Wu, W. Feng, H. L. Sun, S. Lu, Semicond. Sci. Technol. 27 (2012) 045003.

[144] F. A. Khaja, B. Colombeau, T. Thanigaivelan, D. Ramappa, T. Henry, Appl. Phys. Lett. 100 (2012) 112102. 
[145] B. Colombeau, B. Guo, H.-J. Gossmann, F. Khaja, N. Pradhan, A. Waite, K. V. Rao, C. Thomidis, Kyu-Ha Shim, T. Henry, N. Variam, Phys. Status Solidi A 211 (2014) 101.

[146] F. F. Morehead, B. L. Crowder, R. S. Title, J. Appl. Phys. 43 (1972) 1112.

[147] H. Onoda, Y. Nakashima, T. Nagayama, S. Sakai, Proc. 13th Int. Workshop Junction Technology, (2013) 66.

[148] H. Onoda, W. Mizubayashi, Y. Nakashima, M. Masahara, Proc. 14th Int. Workshop Junction Technology, (2014) 126.

[149] B. S. Wood, F. A. Khaja, B. P. Colombeau, S. Sun, A. Waite, M. Jin, H. Chen, O. Chan, T. Thanigaivelan, N. Pradhan, H.-J. L. Gossmann, S. Sharma, V. R. Chavva, M.-P. Cai, M. Okazaki, S. S. Munnangi, C.-N. Ni, W. Suen, C.-P. Chang, A. Mayur, N. Variam, A. D. Brand, ECS Trans. 58 (2013) 249.

[150] W. Mizubayashi, H. Onoda, Y. Nakashima, Y. Ishikawa, T. Matsukawa, K. Endo, Y. Liu, S. O’uchi, J. Tsukada, H. Yamauchi, S. Migita, Y. Morita, H. Ota, M. Masahara, Jpn. J. Appl. Phys. 54 (2015) 04DA06.

[151] O. W. Holland, D. Fathy, J. Narayan, O. S. Oen, Radiat. Eff. Defects Solids 90 (1985) 127.

[152] R. D. Goldberg, J. S. Williams, R. G. Elliman, Nucl. Instrum. Methods Phys. Res. B 106 (1995) 242.

[153] L. Pelaz, R. Duffy, M. Aboy, L. A. Marqués, P. López, I. Santos, B. J. Pawlak, M. J. H. Van Dal, B. Duriez, T. Merelle, G. Doornbos, N. Collaert, L. Witters, R. Rooyackers, W. Vandervorst, M. Jurczak, M. Kaiser, R. G. R. Weemaes, J. G. M. van Berkum, P. Breimer, R. J. P. Lander, IEDM Tech. Dig. (2008) 535-538.

[154] V. C. Venezia, L. Pelaz, H. J. L. Gossmann, A. Agarwal, T. E. Haynes, Phys. Rev. B 69 (2004) 125215.

[155] J. Kedzierski, M. Ieong, E. Novak, T. S. Kanarsky, Y. Zhang, D. Boyd, D. Fried, H.-S. Philip Wong, IEEE Trans. Electron Devices 50 (2003) 952.

[156] N. Collaert, A. De Keersgieter, A. Dixit, I. Ferain, L.-S. Lai, D. Lenoble, A. Mercha, A. Nackaerts, B. J. Pawlak, R. Rooyackers, T. Schulz, K. T. San, N. J. Son, M. J. H. Van Dal, P. Verheyen, K. von Arnim, L. Witters, K. De Meyer, S. Biesemans, M. Jurczak, Solid-State Electron. 52 (2008) 1291.

[157] V. Subramanian, A. Mercha, B. Parvais, J. Loo, C. Gustin, M. Dehan, N. Collaert, M. Jurczak, G. Groeseneken, W. Sansen, S. Decoutere, Solid-State Electron. 51 (2007) 551. 
[158] H. J. L. Gossmann, A. Agarwal, T. Parrill, L. M. Rubin, J. M. Poate, IEEE Trans. Nanotechnology 2 (2003) 285.

[159] Y. Sasaki, K. Okashita, K. Nakamoto, T. Kitaoka, B. Mizuno, M. Ogura, IEDM Tech. Dig. (2008) 917-920.

[160] G. Zschätzsch, Y. Sasaki, S. Hayashi, M. Togo, T. Chiarella, A. K. Kambham, J. Mody, B. Douhard, N. Horiguchi, B. Mizuno, M. Ogura, W. Vandervorst, IEEE International Electron Devices Meeting (IEDM) (2011) 35.6.1-35.6.4.

[161] J. W. Lee, Y. Sasaki, M. J. Cho, M. Togo, G. Boccardi, R. Ritzenthaler, G. Eneman, T. Chiarella, S. Brus, N. Horiguchi, G. Groeseneken, A. Thean, Appl. Phys. Lett. 102 (2013) 223508.

[162] J. -M. Lee, K. H. Cho, D-W. Kim, I. Chung, Jpn. J. Appl. Phys. 55 (2016) 04ED17.

[163] N. D. Nguyen, E. Rosseel, S. Takeuchi, J.-L. Everaert, L. Yang, J. Goossens, A. Moussa, T. Clarysse, O. Richard, H. Bender, S. Zaima, A. Sakai, R. Loo, J. C. Lin, Vandervorst, M. Caymax, Thin Solid Films 518 (2010) S48.

[164] W. Vandervorst, J. L. Everaert, E. Rosseel, M. Jurczak, T. Hoffman, P. Eyben, J. Mody, G. Zschaetzsch, S. Koelling, M. Gilbert, T. Poon, J. del Eyben, M. Foad, R. Duffy, B. J. Pawlak, Ion Implantation Technology AIP Conf. Proc. 1066 (2008) 449-456.

[165] L. Pelaz, L. A. Marqués, M. Aboy, I. Santos, P. López, Ray Duffy, J. Vac. Sci. Technol. B 28 (2010) C1A1.

[166] R. Duffy, G. Curatola, B. J. Pawlak, G. Doornbos, K. van der Tak, P. Breimer, J. G. M. van Berkum, F. Roozeboom, J. Vac. Sci. Technol. B 26 (2008) 402.

[167] A. K. Kambham, J. Mody, M. Gilbert, S. Koelling, W. Vandervorst, Ultramicroscopy 111 (2011) 535.

[168] D. Lenoble, K.G. Anil, A. De Keersgieter, P. Eybens, N. Collaert, R. Rooyackers, S. Brus, P. Zimmerman, M. Goodwin, D. Vanhaeren, W. Vandervorst, S. Radovanov, L. Godet, C. Cardinaud, S. Biesemans, T. Skotnicki, M. Jurczak, "Enhanced performance of PMOS MUGFET via integration of conformal plasmadoped source/drain extensions,” Symp. on VLSI Tech. Dig., pp. 212-213, 2006.

[169] S. I. Garduño, A. Cerdeira, M. Estrada, J. Alvarado, V. Kilchytska, D. Flandre, J. Appl. Phys. 113 (2013) 124507.

[170] L. Pelaz, L. A. Marqués, M. Aboy, P. López, I. Santos, R. Duffy, IEDM Tech. Dig. (2009) 479. 
[171] M. Aboy, L. Pelaz, E. Bruno, S. Mirabella, S. Boninelli, J. Appl. Phys. 110 (2011) 073525.

[172] M. Aboy, I. Santos, L. Pelaz, L. A. Marqués, P. López, J. Comput. Electron. 13 (2014) 40.

[173] M. Aboy, L. Pelaz, L. A. Marqués, P. López, J. Barbolla, R. Duffy, J. Appl. Phys. 97 (2005) 103520.

[174] F. Cristiano, X. Hebras, N. Cherkashin, A. Claverie, W. Lerch, S. Paul, Appl. Phys. Lett. 83 (2003) 5407.

[175] O. Cojocaru-Mirédin, F. Cristiano, P.-F. Fazzini, D. Mangelinck, D. Blavette, Thin Solid Films 534 (2013) 62.

[176] D. De Salvador, E. Napolitani, G. Bisognin, A. Carnera, E. Bruno, S. Mirabella, G. Impellizzeri, F. Priolo, Appl. Phys. Lett. 87 (2005) 221902.

[177] S. Boninelli, S. Mirabella, E. Bruno, F. Priolo, F. Cristiano, A. Claverie, D. De Salvador, G. Bisognin, E. Napolitani, Appl. Phys. Lett. 91 (2007) 031905.

[178] J. Mody, A.K. Kambham, G. Zschätzsch, P. Schatzer, T. Chiarella, N.Collaert, L. Witters, M. Jurczak, N. Horiguchi, M. Gilbert, P. Eyben, S. Kölling, A. Schulze, TY. Hoffmann, W. Vandervorst, Symp. on VLSI Tech.-Dig. of Tech. Pap. (2010) 195-196.

[179] R. Lindsay, B. Pawlak, J. Kittl, K. Henson et al., Mater. Res. Soc. Symp. Proc. 765 (2003) 261-266.

[180] C. Wang, S. Tang, K. Han, H. Persing, H. Maynard, S. Salimian, 20th Int. Conf. on Ion Implantation Technology (2014).

[181] Y. Sasaki, International Workshop on Junction Technology (IWJT) (2010) 1-4.

[182] D. Raj, H. Persing, S. Salimian, K. Lacey, S. Qin., J. Y. Hu., A. McTeer, Ion Implantation Technology AIP Conf. Proc. 1496 (2012) 67-70.

[183] S. Qin, K. Brumfield, L. J. Liu, Y. J. Hu, A. McTeer, W. H. Hsu, M. Wang, Ion Implantation Technology AIP Conf. Proc. 1496 (2012) 380-385.

[184] A. La Magna, G. Fisicaro, G. Nicotra, Y. Spiegel, F. Torregrosa, Phys. Status Solidi C 11 (2014) 109.

[185] A. Burenkov, J. Lorenz, Y. Spiegel, F. Torregrosa, 2015 Int. Conf. on Simulation of Semiconductor Processes and Devices (SISPAD) (2015) 218-221.

[186] N. Zographos, A. Tsibizov, C. Zechner, 20th International Conference on Ion Implantation Technology (2014) 1-6. 